REPRESENTATION THEORY

An Electronic Journal of the American Mathematical Society

Volume 3, Pages 223-249 (August 16, 1999)

S $1088-4165(99) 00056-4$

\title{
THE FINE STRUCTURE OF TRANSLATION FUNCTORS
}

\author{
KAREN GÜNZL
}

\begin{abstract}
Let $E$ be a simple finite-dimensional representation of a semisimple Lie algebra with extremal weight $\nu$ and let $0 \neq e \in E_{\nu}$. Let $M(\tau)$ be the Verma module with highest weight $\tau$ and $0 \neq v_{\tau} \in M(\tau)_{\tau}$. We investigate the projection of $e \otimes v_{\tau} \in E \otimes M(\tau)$ on the central character $\chi(\tau+\nu)$. This is a rational function in $\tau$ and we calculate its poles and zeros. We then apply this result in order to compare translation functors.
\end{abstract}

\section{Contents}

1. Introduction

2. Filtration of $E \otimes M(\tau)$

3. The fine structure of $E \otimes M(\tau)$

3.1. Algebraicity of $f_{\nu}$

3.2. Poles of $f_{\nu}$. Theorem 1 and proof

3.3. Proof of Lemmas $6,7,8$, and 9

4. The triangle function $\Delta$

4.1. Preliminaries

4.2. Definition of $\Delta$

4.3. Uncanonical definition of $\Delta$

5. Bernstein's relative trace and a special case of $\Delta$

5.1. The relative trace

5.2. The special case $\Delta\left(-\nu, \nu ; w_{0}\right)$

5.3. Proof of Theorem 2

5.4. The case $M(\tau) \rightarrow T_{\tau+\nu}^{\tau} T_{\tau}^{\tau+\nu} M(\tau) \rightarrow M(\tau)$

6. Calculation of $\Delta$

7. Outlook

7.1. Identities

7.2. Generalizations

References

\section{INTRODUCTION}

Let $k$ be a field of characteristic zero and let $\mathfrak{g}$ be a split semisimple Lie algebra over $k$. Then $\mathfrak{h} \subset \mathfrak{b} \subset \mathfrak{g}$ denote a Cartan and a Borel subalgebra, $\mathfrak{U}=\mathfrak{U}(\mathfrak{g})$ the universal enveloping algebra and $\mathfrak{Z} \subset \mathfrak{U}$ its center. We set $R^{+} \subset R \subset \mathfrak{h}^{*}$ to be the roots of $\mathfrak{b}$ and of $\mathfrak{g}$. Let $\mathfrak{n}\left(\right.$ resp. $\mathfrak{n}^{-}$) be the sum of the positive (negative) weight

Received by the editors September 2, 1998 and, in revised form, July 19, 1999.

1991 Mathematics Subject Classification. Primary 17B10.

Partially supported by EEC TMR-Network ERB FMRX-CT97-0100.

(C)1999 American Mathematical Society 
spaces and denote by $P^{+} \subset P \subset \mathfrak{h}^{*}$ the set of dominant and the lattice of integral weights respectively. Let $\mathcal{W}$ be the Weyl group.

A $\mathfrak{g}$-module $M$ is called $\mathfrak{Z}$-finite, if $\operatorname{dim}_{k}(\mathfrak{Z} m)<\infty$ for all $m \in M$. Every $\mathfrak{Z}$ finite module $M$ splits under the operation of the center $\mathfrak{Z}$ into a direct sum of submodules $M=\bigoplus_{\chi \in \operatorname{Max} \mathfrak{Z}} M_{\chi}$. Here $\chi$ runs over the maximal ideals in the center and $M_{\chi} \in \mathcal{M}^{\infty}(\chi)$, where

$$
\mathcal{M}^{\infty}(\chi):=\left\{M \mid \text { for all } m \in M \text { there exists } n \in \mathbb{N} \text { such that } \chi^{n} m=0\right\} .
$$

We denote the projection onto the central character $\chi$ by $\operatorname{pr}_{\chi}: M \mapsto M_{\chi}$. By means of the Harish-Chandra homomorphism [Di, 7.4] $\xi: \mathfrak{Z} \rightarrow S(\mathfrak{h})$ (normalized by $z-\xi(z) \in \mathfrak{U} \mathfrak{n} \forall z \in \mathfrak{Z}$ ) we assign to each weight $\tau \in \mathfrak{h}^{*}$ its central character $\chi(\tau)$ defined by $\chi_{\tau}(z):=(\xi(z))(\tau) \forall z \in \mathfrak{Z}$.

Let now $\nu \in P$ be an integral weight and denote by $E:=E(\nu)$ the irreducible finite-dimensional $\mathfrak{g}$-module with highest weight in $\mathcal{W} \nu$. It is known that for each $\mathfrak{Z}$-finite module $M$ the tensor product $E \otimes M$ is again $\mathfrak{Z}$-finite (see [Ko]). If we now tensor a module $M \in \mathcal{M}^{\infty}(\chi(\tau))$ with $E$ and then project onto the central character $\chi(\tau+\nu)$, we obtain the translation functor

$$
\begin{aligned}
T_{\tau}^{\tau+\nu}: \mathcal{M}^{\infty}(\chi(\tau)) & \longrightarrow \mathcal{M}^{\infty}(\chi(\tau+\nu)) \\
M & \mapsto \operatorname{pr}_{\chi(\tau+\nu)}(E \otimes M) .
\end{aligned}
$$

In the present paper we investigate the fine structure of translation functors. Namely, take for $M$ the Verma module $M(\tau):=\mathfrak{U}(\mathfrak{g}) \otimes_{\mathfrak{U}(\mathfrak{b})} k_{\tau}$ with its highest weight vector $v_{\tau}:=1 \otimes 1 \in M(\tau)_{\tau}$ and then identify for all $\tau \in \mathfrak{h}^{*}$ :

$$
\begin{array}{ccc}
M(\tau) & \stackrel{\sim}{\longrightarrow}\left(\mathfrak{n}^{-}\right) \\
u v_{\tau} & \mapsto & u .
\end{array}
$$

We choose a fixed extremal weight vector $e_{\nu} \in E_{\nu}$ and define the map

$$
\begin{aligned}
& f_{\nu}: \mathfrak{h}^{*} \longrightarrow \quad E \otimes \mathfrak{U}\left(\mathfrak{n}^{-}\right) \\
& \tau \mapsto \operatorname{pr}_{\chi(\tau+\nu)}\left(e_{\nu} \otimes v_{\tau}\right) \text {. }
\end{aligned}
$$

Here we identify $\operatorname{pr}_{\chi(\tau+\nu)}\left(e_{\nu} \otimes v_{\tau}\right) \in E \otimes M(\tau)$ with its image in $E \otimes \mathfrak{U}\left(\mathfrak{n}^{-}\right)$. The image of the map $f_{\nu}$ is then contained in the finite-dimensional $\nu$-weight space of $E \otimes \mathfrak{U}\left(\mathfrak{n}^{-}\right)$, and we may thus regard $f_{\nu}$ as a map between varieties. In general however, $f_{\nu}$ is not a morphism.

Let $\rho:=1 / 2 \sum_{\alpha \in R^{+}} \alpha$ denote the half sum of positive roots, $\alpha^{\vee}$ the co-root of $\alpha$ and set

$$
\mathcal{N}_{\nu}:=\left\{\left(\alpha, m_{\alpha}\right) \in R^{+} \times \mathbb{Z} \mid-\left\langle\rho, \alpha^{\vee}\right\rangle \leq m_{\alpha}<-\left\langle\nu+\rho, \alpha^{\vee}\right\rangle\right\} .
$$

Then the set

$$
\mathcal{H}_{\nu}:=\bigcup_{(\alpha, m) \in \mathcal{N}_{\nu}}\left\{\tau \in \mathfrak{h}^{*} \mid\left\langle\tau, \alpha^{\vee}\right\rangle=m\right\}
$$

is a finite family of hyperplanes and therefore Zariski closed. We will show at first that $f_{\nu}$ is a morphism of varieties on the complement of $\mathcal{H}_{\nu} \cup \mathcal{S}$, where $\mathcal{S} \subset \mathfrak{h}^{*}$ is a suitable Zariski closed subset of codimension $\geq 2$. More precisely, $\mathcal{S}$ consists of intersections of finitely many hyperplanes. 
Define for all roots $\alpha \in R$ and for all $m \in \mathbb{Z}$ the polynomial function $H_{\alpha, m}$ on $\mathfrak{h}^{*}$ by

$$
H_{\alpha, m}(\tau):=\left\langle\tau, \alpha^{\vee}\right\rangle-m \text { for all } \tau \in \mathfrak{h}^{*} .
$$

If we then set $\delta_{\nu}:=\prod_{(\alpha, m) \in \mathcal{N}_{\nu}} H_{\alpha, m}$, we obtain $\mathcal{H}_{\nu}=\left\{\tau \in \mathfrak{h}^{*} \mid \delta_{\nu}(\tau)=0\right\}$. A central result of this paper will be the following

Theorem. There exists a morphism of varieties $G: \mathfrak{h}^{*} \rightarrow\left(E \otimes \mathfrak{U}\left(\mathfrak{n}^{-}\right)\right)_{\nu}$, such that the set of zeros of $G$ has codimension $\geq 2$ and such that $G$ equals $\delta_{\nu} f_{\nu}$ on $\mathfrak{h}^{*}-\left(\mathcal{H}_{\nu} \cup \mathcal{S}\right)$.

This means that the map $f_{\nu}$ has a pole of order 1 along each of the hyperplanes $\left\langle\tau, \alpha^{\vee}\right\rangle=m$ with $(\alpha, m) \in \mathcal{N}_{\nu}$ and outside of these hyperplanes it is a non-vanishing morphism of varieties except on a set of codimension $\geq 2$.

We remark that Kashiwara [Ka, Thm. 1.7] has also determined these poles (for universal Verma modules) in the special case of dominant $\tau$ and antidominant $\nu$, i.e. for $\nu$ the lowest weight of $E$. He then used this result to calculate $b$-functions on the flag manifold.

Etingof and Styrkas [ES] have considered a slightly different situation in connection with intertwiners: Instead of looking at the element $\operatorname{pr}_{\chi(\tau+\nu)}\left(e_{\nu} \otimes v_{\tau}\right)$ which for generic $\tau$ is a generator for the Verma module $M(\tau+\nu)$ seen as a submodule of $E \otimes M(\tau)$, consider rather a different generator $w_{\tau+\nu}$ given by $w_{\tau+\nu}:=e_{\nu} \otimes v_{\tau}+R$, where $R \in E \otimes \mathfrak{U}\left(\mathfrak{n}^{-}\right) \mathfrak{n}^{-} v_{\tau}$. By the above theorem we can then express $\operatorname{pr}_{\chi(\tau+\nu)}\left(e_{\nu} \otimes v_{\tau}\right)$ as $\left(\delta_{\nu}(\tau)\right)^{-1} a_{\nu}(\tau) w_{\tau+\nu}$, where $a_{\nu}$ is a polynomial in $\mathfrak{h}^{*}$ and its zeros contain the poles of the coefficient functions occurring in $R$. For $\nu=0$, not necessarily an extremal weight of $E$, these poles (i.e. the poles of $w_{\tau+\nu}$ ) have been computed in $[\mathrm{ES}]$ in terms of the determinant of the Shapovalov form. Etingof and Varchenko later used this result to establish a Hopf-algebroid structure on a certain (rational) exchange quantum group [EV, Ch. 5].

In Chapter 4 we introduce the so-called triangle functions $\Delta(\mu, \nu ; x)(\tau)$ for integral weights $\mu$ and $\nu$ and $x \in \mathcal{W}$. These are rational functions on $\mathfrak{h}^{*}$, which measure in a subtle way the relation between the two translation functors $T_{\tau+\nu}^{\tau+\nu+\mu} \circ T_{\tau}^{\tau+\nu}$ and $T_{\tau}^{\tau+\nu+\mu}$ by first applying them to the Verma modules $M(\tau)$ and $M(x \cdot \tau)$ and then identifying the results with $M(\tau+\nu+\mu)$ and $M(x \cdot(\tau+\nu+\mu))$ respectively.

This construction is the characteristic zero analogue of a function introduced by Andersen, Jantzen and Soergel [AJS, Ch. 11]. In fact, the motivation for the present work was to understand better some of the more technical chapters in [AJS] and in particular realizing the poles and zeros of $\Delta$ in a different way. In order to do this we make use of the maps $f_{\nu}$ for suitable integral weights $\nu$ and can thus write $\Delta$ as a product of the corresponding $\delta_{\nu}$ 's. This is done in Chapter 6 (Lemma 11). If we set now $\bar{\alpha}(\lambda):=1$ for $\left\langle\lambda, \alpha^{\vee}\right\rangle\left\langle 0\right.$ and $\bar{\alpha}(\lambda):=0$ for $\left\langle\lambda, \alpha^{\vee}\right\rangle \geq 0$, we then obtain

Theorem. Let $\nu, \mu \in P$ be integral weights and $x \in \mathcal{W}$. Then

$$
\Delta(\mu, \nu ; x)(\tau-\rho)=c \prod_{\substack{\alpha \in R^{+} \\ \text {with } x \alpha \in R^{-}}} \frac{\left\langle\tau, \alpha^{\vee}\right\rangle^{\bar{\alpha}(\nu)}\left\langle\tau+\nu, \alpha^{\vee}\right\rangle^{\bar{\alpha}(\mu)}\left\langle\tau+\nu+\mu, \alpha^{\vee}\right\rangle^{\bar{\alpha}(\nu+\mu)}}{\left\langle\tau, \alpha^{\vee}\right\rangle^{\bar{\alpha}(\nu+\mu)}\left\langle\tau+\nu, \alpha^{\vee}\right\rangle^{\bar{\alpha}(\nu)}\left\langle\tau+\nu+\mu, \alpha^{\vee}\right\rangle^{\bar{\alpha}(\mu)}}
$$

for a constant $c \in k^{\times}$independent of $\tau, \nu, \mu$ and $x$. 
Bernstein defined in [Be] the so-called relative trace $\operatorname{tr}_{E}$ for a finite-dimensional vector space $E$ and this function is related to the special case $\Delta\left(-\nu, \nu ; w_{0}\right)$, where $\nu$ is dominant and $w_{0}$ is the longest element of $\mathcal{W}$. This is explained in Chapter 5. By Bernstein's explicit formula for $\operatorname{tr}_{E}$ - or alternatively by [Ka, Thm. 1.9] —we obtain

Corollary. Let $\nu \in P^{+}$be dominant and $\tau \in \mathfrak{h}^{*}$ generic, i.e. $\left\langle\tau, \alpha^{\vee}\right\rangle \notin \mathbb{Z}$ for all $\alpha \in R$. Then

$$
\Delta\left(-\nu, \nu ; w_{0}\right)(\tau)=\prod_{\alpha \in R^{+}} \frac{\left\langle\tau+\nu+\rho, \alpha^{\vee}\right\rangle}{\left\langle\tau+\rho, \alpha^{\vee}\right\rangle}
$$

\section{Filtration of $E \otimes M(\tau)$}

In the following let $\nu \in P$ be a fixed integral weight. Then let $\nu_{1}, \ldots, \nu_{n}$ be the multiset of weights of $E=E(\nu)$, i.e. with multiplicities, and let $\left(e_{i}\right)_{1 \leq i \leq n}$ be a basis of weight vectors of $E$, such that $e_{i} \in E_{\nu_{i}}$ and $\nu_{i}<\nu_{j} \Rightarrow i<j$. Here $\lambda \leq \mu$ for weights $\lambda, \mu \in \mathfrak{h}^{*}$, if $\mu-\lambda=\sum_{\alpha \in R^{+}} n_{\alpha} \alpha$ with $n_{\alpha} \in \mathbb{N}$.

In [BGG1] it was shown that the tensor product $E \otimes M(\tau)$ admits a chain of submodules $N_{i}:=\sum_{j=i}^{n} \mathfrak{U}\left(\mathfrak{n}^{-}\right)\left(e_{j} \otimes v_{\tau}\right)$, such that $N_{i} / N_{i+1} \cong M\left(\tau+\nu_{i}\right)$.

Using this, one can easily construct a slightly coarser filtration, namely a filtration such that the subquotients are each isomorphic to a direct sum of Verma modules with the same highest weight. In order to do this, let $\mu_{1}, \mu_{2}, \ldots, \mu_{m}$ denote the set of weights of $E$ without multiplicities and set $d_{j}:=\operatorname{dim} E_{\mu_{j}}$. We may choose the numbering of the $\nu_{i}$ in such a way that $\nu_{k}=\mu_{j}$ for all $k$ with $d_{1}+\cdots+d_{j-1}<k \leq d_{1}+\cdots+d_{j}$. The weight space $E_{\mu_{j}}$ is then generated by precisely these $e_{k}$. Set $d_{0}:=0$ and define for $1 \leq j \leq m$ :

$$
M_{j}:=\sum_{k=j}^{m} \mathfrak{U}\left(\mathfrak{n}^{-}\right)\left(E_{\mu_{k}} \otimes v_{\tau}\right)=\sum_{k>d_{1}+d_{2}+\cdots d_{j-1}}^{n} \mathfrak{U}\left(\mathfrak{n}^{-}\right)\left(e_{k} \otimes v_{\tau}\right) .
$$

We obtain immediately: The chain $E \otimes M(\tau)=M_{1} \supset \cdots \supset M_{m} \supset 0$ is a filtration of submodules such that $M_{j} / M_{j+1} \cong \bigoplus_{d_{j}} M\left(\tau+\mu_{j}\right)$ and the summands $M\left(\tau+\mu_{j}\right)$ are generated by the vectors $\left(e_{i} \otimes v_{\tau}\right) / M_{j+1}=\operatorname{pr}\left(e_{i} \otimes v_{\tau}\right) / M_{j+1}$, where $e_{i}$ is a vector of weight $\mu_{j}$.

Lemma 1. For $1 \leq j \leq m$ let $s_{j} \in \mathfrak{Z}$ with $\chi_{\tau+\mu_{j}}\left(s_{j}\right)=0$ and set $z_{i}:=\prod_{j \geq i} s_{j}$. Then for $1 \leq i \leq m$ we get: $z_{i} M_{i}=0$.

In particular, for a weight vector $e_{\mu_{i}} \in E_{\mu_{i}}$ we obtain

$$
z_{i+1}\left(e_{\mu_{i}} \otimes v_{\tau}\right)=z_{i+1} \operatorname{pr}_{\chi\left(\tau+\mu_{i}\right)}\left(e_{\mu_{i}} \otimes v_{\tau}\right) .
$$

Proof. The first statement follows by induction from above and since a central element $z \in \mathfrak{Z}$ operates on the Verma module $M(\lambda)$ by multiplication with the scalar $\chi_{\lambda}(z)$.

By construction of the $M_{i}$ it is clear, that for $e_{\mu_{i}} \otimes v_{\tau} \in M_{i}$ its projections $\operatorname{pr}_{\chi(\tau+\mu)}\left(e_{\mu_{i}} \otimes v_{\tau}\right)$ lie already in $M_{i+1}$ if $\chi(\tau+\mu) \neq \chi\left(\tau+\mu_{i}\right)$. But $e_{\mu_{i}} \otimes v_{\tau}$ equals the sum of its projections and thus the second statement follows from the first.

Note that for the extremal weight $\nu=\nu_{i_{0}}=\mu_{j_{0}}$ its weight space is of dimension 1. Therefore, we can always choose the numbering of the $\nu_{i}$ such that $i>i_{0} \Longleftrightarrow$ $\nu_{i}>\nu_{i_{0}}=\nu$ or equivalently $j>j_{0} \Longleftrightarrow \mu_{j}>\mu_{j_{0}}=\nu$. It then follows immediately that an element of $M_{i_{0}}=M_{j_{0}}$ (of $M_{i_{0}+1}=M_{j_{0}+1}$ resp.) consists only of parts with 
central character $\chi_{\tau+\mu}$ for $\mu \in P(E)$ with $\mu \geq \nu$ ( $\mu>\nu$ resp.). For $e_{\nu} \otimes v_{\tau} \in M_{i_{0}}$ we thus obtain the following special case of Lemma 1.

Lemma 2. For $\mu \in P(E)$ with $\mu>\nu$ let $s_{\mu} \in \mathfrak{Z}$ such that $\chi_{\tau+\mu}\left(s_{\mu}\right)=0$ and set $z:=\prod_{\mu>\nu} s_{\mu}$. Then $z\left(e_{\nu} \otimes v_{\tau}\right)=z \operatorname{pr}_{\chi(\tau+\nu)}\left(e_{\nu} \otimes v_{\tau}\right)$.

\section{The Fine Structure of $E \otimes M(\tau)$}

3.1. Algebraicity of $f_{\nu}$. For $\nu \in P$ fixed, set $E:=E(\nu)$ and let $f_{\nu}$ be the map

$$
\begin{aligned}
f_{\nu}: \mathfrak{h}^{*} & \longrightarrow \\
\tau & \mapsto \operatorname{pr}_{\chi(\tau+\nu)}\left(e_{\nu} \otimes v_{\tau}\right)
\end{aligned}
$$

where we identify $E \otimes \mathfrak{U}\left(\mathfrak{n}^{-}\right) \cong E \otimes M(\tau)$ for all $\tau \in \mathfrak{h}^{*}$. We will first construct a Zariski open set $\mathcal{U} \subset \mathfrak{h}^{*}$ such that $f_{\nu}$ restricted to $\mathcal{U}$ is a morphism of varieties. In this case we will also say that $f_{\nu}$ is algebraic on $\mathcal{U}$.

Before we go on, we need some more notation: The dot-operation of the Weyl group on $\mathfrak{h}^{*}$ has fixed point $-\rho=-1 / 2 \sum_{\alpha \in R^{+}} \alpha$ and is defined by $w \cdot \lambda:=$ $w(\lambda+\rho)-\rho$. Let $\mathcal{P}\left(\mathfrak{h}^{*}\right)$ denote the set of polynomial functions on $\mathfrak{h}^{*}$ and $\mathcal{P}\left(\mathfrak{h}^{*}\right)^{\mathcal{W}}$. the $(\mathcal{W} \cdot)$-invariants. We define the operator

$$
\text { sym : } \mathcal{P}\left(\mathfrak{h}^{*}\right) \longrightarrow \mathcal{P}\left(\mathfrak{h}^{*}\right)^{\mathcal{W}}
$$

by $(\operatorname{sym} s)(\lambda):=\prod_{x \in \mathcal{W}} s(x \cdot \lambda) \quad$ for all $\lambda \in \mathfrak{h}^{*}, s \in \mathcal{P}\left(\mathfrak{h}^{*}\right)$.

For any $\mu \in P(E)$ with $\mu>\nu$ we now choose an element $h_{\mu} \in \mathfrak{h}, h_{\mu} \neq 0$ and define maps $H_{\mu}: \mathfrak{h}^{*} \times \mathfrak{h}^{*} \longrightarrow k$ by

$$
H_{\mu}(\lambda, \tau):=\left\langle\lambda-\tau-\mu, h_{\mu}\right\rangle \quad \text { for all } \lambda, \tau \in \mathfrak{h}^{*} .
$$

If we fix $\tau \in \mathfrak{h}^{*}$, the map $H_{\mu}(-, \tau): \mathfrak{h}^{*} \longrightarrow k$ is then a polynomial function on $\mathfrak{h}^{*}$ and its kernel is obviously a hyperplane in $\mathfrak{h}^{*}$. We then define a $(\mathcal{W} \cdot)$-invariant polynomial function $p_{\tau}$, depending also on the choice of the elements $h_{\mu}$, by

$$
p_{\tau}:=\prod_{\mu \in P(E), \mu>\nu} \operatorname{sym} H_{\mu}(-, \tau) .
$$

By means of the Harish-Chandra isomorphism [Di, 7.4] $\xi: \mathfrak{Z} \stackrel{\sim}{\longrightarrow} \mathcal{P}\left(\mathfrak{h}^{*}\right)^{\mathcal{W}}$, there exist central elements $s_{\mu} \in \mathfrak{Z}$, such that $\xi\left(s_{\mu}\right)=\operatorname{sym} H_{\mu}(-, \tau)$. For $z_{\tau}:=\prod_{\mu>\nu} s_{\mu}$ we have $\xi\left(z_{\tau}\right)=p_{\tau}$. Define now the map

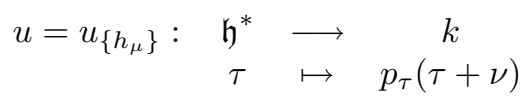

and set $\mathcal{U}_{\left\{h_{\mu}\right\}}:=\left\{\tau \in \mathfrak{h}^{*} \mid u(\tau) \neq 0\right\} \subset \mathfrak{h}^{*}$.

Lemma 3. The map $f_{\nu}$ restricted to $\mathcal{U}_{\left\{h_{\mu}\right\}}$ is a morphism of varieties and for all $\tau \in \mathcal{U}_{\left\{h_{\mu}\right\}}$ we have $f_{\nu}(\tau)=(u(\tau))^{-1} z_{\tau}\left(e_{\nu} \otimes v_{\tau}\right)$.

Proof. We know $\chi_{\tau+\mu}\left(s_{\mu}\right)=\left(\xi\left(s_{\mu}\right)\right)(\tau+\mu)=\left(\operatorname{sym} H_{\mu}(-, \tau)\right)(\tau+\mu)=0$ and by Lemma 2 we conclude $z_{\tau}\left(e_{\nu} \otimes v_{\tau}\right)=z_{\tau} \operatorname{pr}_{\chi(\tau+\nu)}\left(e_{\nu} \otimes v_{\tau}\right)$.

Let now $\tau \in \mathcal{U}_{\left\{h_{\mu}\right\}}$, i.e. $u(\tau) \neq 0$. This means, that for all $w \in \mathcal{W}$ and for all $\mu \in P(E)$ with $\mu>\nu$ we have $\left\langle w \cdot(\tau+\nu)-\tau-\mu, h_{\mu}\right\rangle \neq 0$ and in particular $w \cdot(\tau+\nu) \neq \tau+\mu$ or equivalently $\chi(\tau+\nu) \neq \chi(\tau+\mu) \forall \mu \in P(E), \mu>\nu$. This implies, that already the projection $\operatorname{pr}_{\chi(\tau+\nu)}\left(e_{\nu} \otimes v_{\tau}\right) \in E \otimes M(\tau)$ generates a Verma module $M(\tau+\nu)$. A central element $z$ operates on this by multiplication with the 
scalar $(\xi(z))(\tau+\nu)=u(\tau)$. We therefore get $z_{\tau}\left(e_{\nu} \otimes v_{\tau}\right)=z_{\tau} \operatorname{pr}_{\chi(\tau+\nu)}\left(e_{\nu} \otimes v_{\tau}\right)=$ $u(\tau) f_{\nu}(\tau)$ and the above equation follows.

We have yet to show that $f_{\nu}$ is algebraic on $\mathcal{U}_{\left\{h_{\mu}\right\}}$. By construction of $z_{\tau}$ it is clear that $z_{\tau}$ depends algebraically on $\tau$ for all $\tau \in \mathfrak{h}^{*}$. Also the map $\tau \mapsto$ $z_{\tau}\left(e_{\nu} \otimes v_{\tau}\right) \in E \otimes \mathfrak{U}\left(\mathfrak{n}^{-}\right)$is a morphism on $\mathfrak{h}^{*}$ and $1 / u$ is per definition algebraic on $\mathcal{U}_{\left\{h_{\mu}\right\}}$. Thus $f_{\nu}$ restricted to $\mathcal{U}_{\left\{h_{\mu}\right\}}$ is a morphism.

Example. Let $\nu \in P(E)$ be an extremal and dominant weight. For all $\mu \in P(E)$ with $\mu \neq \nu$ we then have $\mu<\nu$ and we may choose $z_{\tau}:=1 \in \mathfrak{Z}$ for all $\tau \in \mathfrak{h}^{*}$. The above lemma then implies $\mathcal{U}_{\left\{h_{\mu}\right\}}=\mathfrak{h}^{*}$, the map $f_{\nu}$ is a morphism on $\mathfrak{h}^{*}$ and $f_{\nu}(\tau)=e_{\nu} \otimes v_{\tau}$ for all $\tau$.

Lemma 3 implies that $f_{\nu}$ is algebraic on $\mathcal{U}:=\bigcup \mathcal{U}_{\left\{h_{\mu}\right\}}$, where the union is taken over all possible choices for $\left\{h_{\mu} \mid \mu \in P(E), \mu>\nu\right\}$. If we set

$$
\mathcal{A}:=\left\{\tau \in \mathfrak{h}^{*} \mid u(\tau)=p_{\tau}(\tau+\nu)=0 \text { for all choices of }\left\{h_{\mu}\right\}\right\},
$$

we can write $\mathcal{U}$ as $\mathcal{U}=\mathfrak{h}^{*}-\mathcal{A}$. Since

$$
p_{\tau}(\tau+\nu)=\prod_{\mu \in P(E), \mu>\nu} \prod_{w \in \mathcal{W}}\left\langle w \cdot(\tau+\nu)-\tau-\mu, h_{\mu}\right\rangle
$$

we know that $\tau$ is in $\mathcal{A}$ if and only if there exists a $w \in \mathcal{W}$ and a $\mu \in P(E)$ with $\mu>\nu$, such that $w \cdot(\tau+\nu)=\tau+\mu$. Thus we obtain

$$
\mathcal{A}=\bigcup_{\substack{\mu \in P(E), \mu>\nu \\ w \in \mathcal{W}}}\left\{\tau \in \mathfrak{h}^{*} \mid w \cdot(\tau+\nu)=\tau+\mu\right\} .
$$

Let us examine more closely the sets on the right hand side: For $w=s_{\alpha}, \alpha \in R$, we get $\left\{\tau \in \mathfrak{h}^{*} \mid\left\langle\tau, \alpha^{\vee}\right\rangle \alpha=\nu-\mu-\left\langle\nu+\rho, \alpha^{\vee}\right\rangle \alpha\right\}$, which implies that this set is nonempty if and only if there exists a weight $\mu=\nu-\left\langle\tau+\nu+\rho, \alpha^{\vee}\right\rangle \alpha$ in the $\alpha$-string through $\nu$, such that $\mu \in P(E)$ and $\mu>\nu$. Since $\nu$ was an extremal weight, it is either the greatest or the smallest weight in this $\alpha$-string and hence such a $\mu$ exists only if $\left\langle\nu, \alpha^{\vee}\right\rangle<0$ for an $\alpha \in R^{+}$. In this case all $\mu_{(n)}:=\nu+n \alpha$ for $1 \leq n \leq-\left\langle\nu, \alpha^{\vee}\right\rangle$ are weights of $E$ with $\mu_{(n)} \geq \nu$. Comparing this with $\mu=\nu-\left\langle\nu+\rho+\tau, \alpha^{\vee}\right\rangle \alpha$ we obtain as a condition for $\tau$, precisely $-\left\langle\rho, \alpha^{\vee}\right\rangle \leq\left\langle\tau, \alpha^{\vee}\right\rangle \leq-\left\langle\nu+\rho, \alpha^{\vee}\right\rangle-1$ for an $\alpha \in R^{+}$.

Set $\mathcal{W}^{0}:=\left\{w \in \mathcal{W} \mid w \neq s_{\alpha}\right.$ for an $\left.\alpha \in R\right\}$. For

$$
\begin{aligned}
\mathcal{N}_{\nu} & :=\left\{\left(\alpha, m_{\alpha}\right) \in R^{+} \times \mathbb{Z} \mid-\left\langle\rho, \alpha^{\vee}\right\rangle \leq m_{\alpha}<-\left\langle\nu+\rho, \alpha^{\vee}\right\rangle\right\}, \\
\mathcal{H}_{\nu} & :=\bigcup_{(\alpha, m) \in \mathcal{N}_{\nu}}\left\{\tau \in \mathfrak{h}^{*} \mid\left\langle\tau, \alpha^{\vee}\right\rangle=m\right\}, \\
\mathcal{S} & :=\bigcup_{\substack{\mu \in \mathcal{P}(E), \mu>\nu \\
w \in \mathcal{W}^{0}}}\left\{\tau \in \mathfrak{h}^{*} \mid w \cdot(\tau+\nu)=\tau+\mu\right\},
\end{aligned}
$$

we get $\mathcal{A}=\mathcal{H}_{\nu} \cup \mathcal{S}$. Remark that in general $\mathcal{H}_{\nu}$ and $\mathcal{S}$ are not disjoint. We claim that $\operatorname{codim} \mathcal{S} \geq 2$ : for $w \in \mathcal{W}$ and $\mu \in P$ let $\mathcal{S}(w, \mu):=\left\{\tau \in \mathfrak{h}^{*} \mid w \cdot(\tau+\nu)=\tau+\mu\right\}$. Since $\mathcal{S}$ is a finite union of such sets it will be enough to show that $\operatorname{codim} \mathcal{S}(w, \mu) \geq 2$ for $w \in \mathcal{W}^{0}$ and $\mu>\nu$. First rewrite $w \cdot(\tau+\nu)=\tau+\mu$ as $(w-\mathrm{id})(\tau)=\mu-w \cdot \nu$ and note that $\operatorname{codim} \mathcal{S}(w, \mu)=\operatorname{rank}(w-\mathrm{id})=\operatorname{dim} \mathfrak{h}^{*}-m_{w}$, where $m_{w}$ denotes the multiplicity of the eigenvalue 1 of $w$ since $w$ is diagonizable. If we assume now $\operatorname{codim} \mathcal{S}(w, \mu) \leq 1$, then $m_{w}=\operatorname{dim} \mathfrak{h}^{*}$ or $m_{w}=\operatorname{dim} \mathfrak{h}^{*}-1$ which implies either 
$w=e$ or $w=s_{\alpha}$ for some $\alpha \in R$. But for $w \in \mathcal{W}^{0}$ and $\mu>\nu$ this is impossible and we get in this case that $\operatorname{codim} \mathcal{S}(w, \mu)=\operatorname{rank}(w-$ id $) \geq 2$.

This implies also that a set $\mathcal{S}(w, \mu)$ appearing in $\mathcal{S}$ consists of an intersection of at least two integral hyperplanes in $\mathfrak{h}^{*}$.

Using Lemma 3 we obtain

Lemma 4. The map $f_{\nu}$ is algebraic on the complement of $\mathcal{H}_{\nu} \cup \mathcal{S}$.

3.2. Poles of $f_{\nu}$. Theorem 1 and proof. Let now $\delta_{\nu} \in \mathcal{P}\left(\mathfrak{h}^{*}\right)$ be the product of the equations of the hyperplanes in $\mathcal{H}_{\nu}$; that is

$$
\delta_{\nu}(\tau):=\prod_{(\alpha, m) \in \mathcal{N}_{\nu}} H_{\alpha, m}(\tau)=\prod_{\alpha \in R^{+}} \prod_{0 \leq n_{\alpha}<-\left\langle\nu, \alpha^{\vee}\right\rangle}\left(\left\langle\tau+\rho, \alpha^{\vee}\right\rangle-n_{\alpha}\right),
$$

where $H_{\alpha, m}(\tau):=\left\langle\tau, \alpha^{\vee}\right\rangle-m$. We then have $\mathcal{H}_{\nu}=\left\{\tau \in \mathfrak{h}^{*} \mid \delta_{\nu}(\tau)=0\right\}$.

Theorem 1. There exists a morphism of varieties $G: \mathfrak{h}^{*} \longrightarrow\left(E(\nu) \otimes \mathfrak{U}\left(\mathfrak{n}^{-}\right)\right)_{\nu}$, such that the set of zeros of $G$ has codimension $\geq 2$ and such that $G$ equals $\delta_{\nu} f_{\nu}$ on $\mathfrak{h}^{*}-\left(\mathcal{H}_{\nu} \cup \mathcal{S}\right)$.

The proof comes in two parts. In the first part we demonstrate the existence of an algebraic extension $G$ of $\delta_{\nu} f_{\nu}$ on the whole of $\mathfrak{h}^{*}$, in the second we will show that the set of zeros of $G$ has codimension $\geq 2$. It will be useful to introduce the set $\mathcal{S}_{1} \subset \mathfrak{h}^{*}$ of all intersections of hyperplanes in $\mathcal{H}_{\nu}$. So if we set

$$
\mathcal{S}_{1}:=\left\{\tau \in \mathfrak{h}^{*} \mid H_{\alpha, m}(\tau)=0=H_{\beta, n}(\tau) \text { for }(\alpha, m) \neq(\beta, n) \in \mathcal{N}_{\nu}\right\},
$$

then $\mathcal{S} \cup \mathcal{S}_{1}$ is a Zariski closed subset of codimension $\geq 2$. By definition of $\mathcal{S}$ and $\mathcal{S}_{1}$ we obtain immediately

Lemma 5. Let $\tau \in \mathcal{H}_{\nu}-\left(\mathcal{S} \cup \mathcal{S}_{1}\right)$. Then there exists exactly one $\alpha \in R^{+}$, such that $s_{\alpha} \cdot(\tau+\nu)=\tau+\mu_{0}$ for a weight $\mu_{0}$ of $E$ with $\mu_{0}>\nu$, namely $\mu_{0}=\nu-\left\langle\tau+\nu+\rho, \alpha^{\vee}\right\rangle \alpha$. For all $\mu \in P(E)$ with $\mu>\nu$ and $\mu \neq \mu_{0}$ we have $w \cdot(\tau+\nu) \neq \tau+\mu$ for all $w \in \mathcal{W}$.

Proof of Theorem 1. Again let $\mathcal{U}=\mathfrak{h}^{*}-\left(\mathcal{H}_{\nu} \cup \mathcal{S}\right)$ be the set on which $f_{\nu}$ is algebraic. First we claim

(*) For all $\tau_{0} \in \mathfrak{h}^{*}-\left(\mathcal{S} \cup \mathcal{S}_{1}\right)$ there exists a Zariski open neighborhood $\mathcal{U}_{0}$ of $\tau_{0}$ and an algebraic map $G_{0}: \mathcal{U}_{0} \rightarrow\left(E \otimes \mathfrak{U}\left(\mathfrak{n}^{-}\right)\right)_{\nu}$, such that $G_{0}=\delta_{\nu} f_{\nu}$ on $\mathcal{U} \cap \mathcal{U}_{0}$

This statement then implies the existence of an algebraic extension of $\delta_{\nu} f_{\nu}$ locally around every point of $\mathfrak{h}^{*}-\left(\mathcal{S} \cup \mathcal{S}_{1}\right)$. This extension is unique and thus we obtain a global algebraic extension on $\mathfrak{h}^{*}-\left(\mathcal{S} \cup \mathcal{S}_{1}\right)$. Since $\operatorname{codim}\left(\mathcal{S} \cup \mathcal{S}_{1}\right) \geq 2$ we can extend this to a morphism $G$ on the whole of $\mathfrak{h}^{*}$.

Let now $\tau_{0} \in \mathfrak{h}^{*}-\left(\mathcal{S} \cup \mathcal{S}_{1}\right)$.

If $\tau_{0} \notin \mathcal{H}_{\nu}$, then $\tau_{0} \in \mathcal{U}=\mathfrak{h}^{*}-\left(\mathcal{H}_{\nu} \cup \mathcal{S}\right)$ and since $f_{\nu}$ was algebraic on $\mathcal{U}$ (Lemma 4), claim (*) follows with $\mathcal{U}_{0}:=\mathcal{U}$ and $G_{0}:=\delta_{\nu} f_{\nu}$.

Let now $\tau_{0} \in \mathcal{H}_{\nu}$. By assumption we then have $\tau_{0} \in \mathcal{H}_{\nu}-\left(\mathcal{S} \cup \mathcal{S}_{1}\right)$, and Lemma 5 implies that $\left\langle\tau_{0}, \alpha^{\vee}\right\rangle=t_{0} \in \mathbb{Z}$ for exactly one $\alpha \in R^{+}$and $\mu_{0}=\nu-\left\langle\nu+\rho+\tau_{0}, \alpha^{\vee}\right\rangle \alpha$ is a weight of $E$ with $\mu_{0}>\nu$.

Again we construct a set $\mathcal{U}_{\left\{h_{\mu}\right\}}$ with a particular choice of $\left\{h_{\mu}\right\}$. Namely, choose for all weights $\mu \in P(E)$ with $\mu>\nu$ and $\mu \neq \mu_{0}$ elements $h_{\mu} \in \mathfrak{h}$, such that $H_{\mu}\left(w \cdot\left(\tau_{0}+\nu\right), \tau_{0}\right)=\left\langle w \cdot\left(\tau_{0}+\nu\right)-\tau_{0}-\mu, h_{\mu}\right\rangle \neq 0$ for all $w \in \mathcal{W}$. For $\mu_{0}$ however, choose $h_{\mu_{0}}$ such that 
a) $\left\langle\alpha, h_{\mu_{0}}\right\rangle \neq 0$ and

b) $H_{\mu_{0}}\left(w \cdot\left(\tau_{0}+\nu\right), \tau_{0}\right)=\left\langle w \cdot\left(\tau_{0}+\nu\right)-\tau_{0}-\mu_{0}, h_{\mu_{0}}\right\rangle \neq 0$ for all $s_{\alpha} \neq w \in \mathcal{W}$.

Lemma 5 ensures that such a choice of $\left\{h_{\mu}\right\}$ is always possible. For $\tau \in \mathfrak{h}^{*}$ we set again $p_{\tau}:=\prod_{\mu \in P(E), \mu>\nu} \operatorname{sym} H_{\mu}(-, \tau)$, and $\xi^{-1}\left(p_{\tau}\right)=: z_{\tau} \in \mathfrak{Z}$. For $u$ defined by $u(\tau)=p_{\tau}(\tau+\nu)$ we then obtain $\mathcal{U}_{\left\{h_{\mu}\right\}}=\left\{\tau \in \mathfrak{h}^{*} \mid u(\tau) \neq 0\right\}$. According to Lemma 3 the map $f_{\nu}$ restricted to $\mathcal{U}_{\left\{h_{\mu}\right\}}$ is algebraic. Let us have a closer look at the map $u$ : It vanishes along the hyperplane $\operatorname{ker} H_{\alpha, t_{0}}$, because for all $\tau \in \mathfrak{h}^{*}$ we have $H_{\mu_{0}}\left(s_{\alpha} \cdot(\tau+\nu), \tau\right)=-\left\langle\alpha, h_{\mu_{0}}\right\rangle \cdot\left(\left\langle\tau, \alpha^{\vee}\right\rangle-t_{0}\right)=a \cdot H_{\alpha, t_{0}}(\tau)$, where $a:=-\left\langle\alpha, h_{\mu_{0}}\right\rangle \neq 0$ according to assumption a).

All the other hyperplanes along which $u$ vanishes are not equal to ker $H_{\alpha, t_{0}}$ but do at most intersect it. Otherwise, (since $\tau_{0} \in \operatorname{ker} H_{\alpha, t_{0}}$ ) we would have $H_{\mu}\left(w \cdot\left(\tau_{0}+\nu\right), \tau_{0}\right)=0$ for a pair $(\mu, w) \neq\left(\mu_{0}, s_{\alpha}\right)$ which is impossible by our choice of the $h_{\mu}$.

For $\bar{u}: \mathfrak{h}^{*} \rightarrow k$ defined by $\bar{u} H_{\alpha, t_{0}}=u$, it follows by what we just said that $\bar{u}\left(\tau_{0}\right) \neq 0$. With $\mathcal{U}_{0}:=\left\{\tau \in \mathfrak{h}^{*} \mid \bar{u}(\tau) \neq 0\right\}$ we then get

- $\tau_{0} \in \mathcal{U}_{0}$,

- $1 / \bar{u}$ is algebraic on $\mathcal{U}_{0}$,

- $\mathcal{U}_{\left\{h_{\mu}\right\}}=\mathcal{U}_{0}-\operatorname{ker} H_{\alpha, t_{0}}$, thus $\mathcal{U}_{0}=\mathcal{U}_{\left\{h_{\mu}\right\}} \cup \operatorname{ker} H_{\alpha, t_{0}}$,

- $\left(\mathcal{U} \cap \mathcal{U}_{0}\right) \subset \mathcal{U}_{\left\{h_{\mu}\right\}}$, since $\mathcal{U} \cap \operatorname{ker} H_{\alpha, t_{0}}=\emptyset$.

If we define $\bar{\delta}=\bar{\delta}_{\nu}: \mathfrak{h}^{*} \rightarrow k$ by $\bar{\delta} H_{\alpha, t_{0}}=\delta_{\nu}$ and set

$$
\begin{array}{ccc}
G_{0}: \mathcal{U}_{0} & \longrightarrow & E \otimes \mathfrak{U}\left(\mathfrak{n}^{-}\right) \\
\tau & \mapsto \bar{\delta}(\tau)(\bar{u}(\tau))^{-1} z_{\tau}\left(e_{\nu} \otimes v_{\tau}\right),
\end{array}
$$

we know that $G_{0}$ is algebraic on $\mathcal{U}_{0}$. In particular, $G_{0}$ is algebraic on an open neighborhood of $\tau_{0}$. This is now the local algebraic extension of $\delta_{\nu} f_{\nu}$ around $\tau_{0}$ which we were looking for, because according to Lemma 3 we have for all $\tau \in \mathcal{U}_{\left\{h_{\mu}\right\}}$ :

$$
\begin{aligned}
\delta_{\nu}(\tau) f_{\nu}(\tau) & =\delta_{\nu}(\tau)(u(\tau))^{-1} z_{\tau}\left(e_{\nu} \otimes v_{\tau}\right) \\
& =\bar{\delta}_{\nu}(\tau) H_{\alpha, t_{0}}(\tau)\left(H_{\alpha, t_{0}}(\tau)\right)^{-1}(\bar{u}(\tau))^{-1} z_{\tau}\left(e_{\nu} \otimes v_{\tau}\right) \\
& =G_{0}(\tau) .
\end{aligned}
$$

In particular, this equation holds for all $\tau \in\left(\mathcal{U} \cap \mathcal{U}_{0}\right) \subset \mathcal{U}_{\left\{h_{\mu}\right\}}$, i.e. $(*)$.

It remains to show that the set of zeros of $G$ has codimension $\geq 2$.

In order to do this, let $\mathcal{S}_{2}$ be the union of all intersections of hyperplanes in $\mathcal{H}_{\nu}$ with any other integral hyperplanes, that is

$$
\mathcal{S}_{2}:=\mathcal{S}_{1} \cup\left\{\tau \in \mathcal{H}_{\nu} \mid\left\langle\tau, \alpha^{\vee}\right\rangle=m \in \mathbb{Z} \text { for a pair }(\alpha, m) \notin \mathcal{N}_{\nu}\right\} .
$$

We claim

$(* *) \mathcal{K}:=\left\{\tau \in \mathfrak{h}^{*} \mid G(\tau)=0\right\} \subset\left(\mathcal{S} \cup \mathcal{S}_{2}\right)$

and conclude then that $\operatorname{codim} \mathcal{K} \geq 2$. Indeed, by definition the set $\mathcal{S} \cup \mathcal{S}_{2}$ consists of a countable family of intersections of integral hyperplanes. On the other hand, $G$ is algebraic on $\mathfrak{h}^{*}$ and therefore its set of zeros must be Zariski closed. Since a countable family of intersections of integral hyperplanes is Zariski closed only if it is a finite family of such intersections, the set $\mathcal{K}$ must have codimension $\geq 2$.

Let now $\tau_{0} \in \mathfrak{h}^{*}-\left(\mathcal{S} \cup \mathcal{S}_{2}\right)$. We have to show $G\left(\tau_{0}\right) \neq 0$.

If $\tau_{0} \notin \mathcal{H}_{\nu}$, we have $\delta_{\nu}\left(\tau_{0}\right) \neq 0$ and $\tau_{0} \in \mathcal{U}=\mathfrak{h}^{*}-\left(\mathcal{H}_{\nu} \cup \mathcal{S}\right)$. Now $G$ equals $\delta_{\nu} f_{\nu}$ on $\mathcal{U}$ and hence $G\left(\tau_{0}\right)=\delta_{\nu}\left(\tau_{0}\right) f_{\nu}\left(\tau_{0}\right) \neq 0$. 
Let now $\tau_{0} \in \mathcal{H}_{\nu}$. Again by the first part of the proof we know that there is an open neighborhood $\mathcal{U}_{0}$ of $\tau_{0}$, such that $G(\tau)=\bar{\delta}_{\nu}(\tau)(\bar{u}(\tau))^{-1} z_{\tau}\left(e_{\nu} \otimes v_{\tau}\right)$ for all $\tau \in \mathcal{U}_{0}$. Since $\bar{\delta}_{\nu}\left(\tau_{0}\right) \neq 0$ it thus suffices to show

$(* *)^{\prime} z_{\tau_{0}}\left(e_{\nu} \otimes v_{\tau_{0}}\right) \neq 0$.

In order to do this, we will use Lemmas 6 to 9 of the following section. Set $e:=e_{\nu}$ and denote the projection onto the central character $\chi\left(\tau_{0}+\nu\right)$ by $\mathrm{pr}=$ $\operatorname{pr}_{\chi\left(\tau_{0}+\nu\right)}: E \otimes \mathfrak{U}\left(\mathfrak{n}^{-}\right) \rightarrow E \otimes \mathfrak{U}\left(\mathfrak{n}^{-}\right)$.

Since $\tau_{0} \in \mathcal{H}_{\nu}-\left(\mathcal{S} \cup \mathcal{S}_{2}\right)$, there exists exactly one $\alpha \in R^{+}$such that $\left\langle\tau_{0}, \alpha^{\vee}\right\rangle \in \mathbb{Z}$. Therefore, $R_{\tau_{0}}:=\left\{\beta \in R \mid\left\langle\tau_{0}, \beta^{\vee}\right\rangle \in \mathbb{Z}\right\}=\{\alpha,-\alpha\}$ and hence the Weyl group $\mathcal{W}_{\tau_{0}}$ of this root system $R_{\tau_{0}}$ equals $\mathcal{W}_{\tau_{0}}=\left\langle s_{\beta} \mid \beta \in R_{\tau_{0}}\right\rangle=\left\langle s_{\alpha}\right\rangle$. For $\tau_{0}$ and $\mu_{0}=\nu-\left\langle\tau_{0}+\nu+\rho, \alpha^{\vee}\right\rangle \alpha$ we may then apply Lemma 8 and obtain a short exact sequence

$$
\bigoplus_{\operatorname{dim} E_{\mu_{0}}} M\left(s_{\alpha} \cdot\left(\tau_{0}+\nu\right)\right) \hookrightarrow \operatorname{pr}\left(E \otimes M\left(\tau_{0}\right)\right) \rightarrow L\left(\tau_{0}+\nu\right)
$$

such that $\operatorname{pr}\left(e \otimes v_{\tau_{0}}\right) / \operatorname{pr}\left(E \otimes M\left(\tau_{0}\right)\right)$ generates the simple module $L\left(\tau_{0}+\nu\right)$. The module $\operatorname{pr}\left(E \otimes M\left(\tau_{0}\right)\right)$ is projective (Lemma 7$)$ and this forces the above short exact sequence to be a nontrivial extension because otherwise, being a direct summand, $L\left(\tau_{0}+\nu\right)$ would be projective too. This contradicts Lemma 7 .

Therefore (see for example [HS, Ch. 3, Lemma 4.1]) we have at least one direct summand $M\left(s_{\alpha} \cdot\left(\tau_{0}+\nu\right)\right)$ of $R_{2}:=\bigoplus_{\operatorname{dim} E_{\mu_{0}}} M\left(s_{\alpha} \cdot\left(\tau_{0}+\nu\right)\right)$, such that the projection pro: $R_{2} \rightarrow M\left(s_{\alpha} \cdot\left(\tau_{0}+\nu\right)\right)$ induces a nontrivial extension

$$
M\left(s_{\alpha} \cdot\left(\tau_{0}+\nu\right)\right) \hookrightarrow \mathrm{PO} \rightarrow L\left(\tau_{0}+\nu\right)
$$

together with a homomorphism pro $: R_{1}:=\operatorname{pr}\left(E \otimes M\left(\tau_{0}\right)\right) \rightarrow$ PO, such that the following diagram commutes (the rows are exact, $\mathrm{PO}$ is a push-out of pro: $R_{2} \rightarrow$ $M\left(s_{\alpha} \cdot\left(\tau_{0}+\nu\right)\right)$ and $R_{2} \hookrightarrow R_{1}$; see for example the dual statement to [HS, Ch. 3 , Lemma 1.3]):

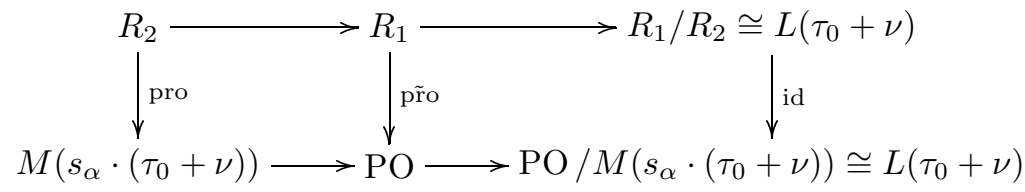

Since the bottom row is a nontrivial extension and since $\mathcal{W}_{\tau_{0}+\nu}=\mathcal{W}_{\tau_{0}}=\left\langle s_{\alpha}\right\rangle$, the push-out $\mathrm{PO}$ is isomorphic to $P\left(\tau_{0}+\nu\right)$, the projective cover of $L\left(\tau_{0}+\nu\right)$ in $\mathcal{O}$ (Lemma 6). In the right loop of the above diagram lies the following element diagram:

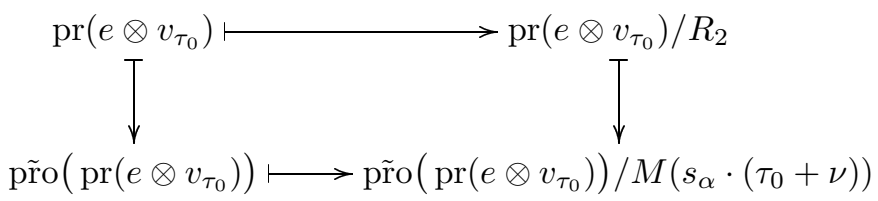

Since $\operatorname{pr}\left(e \otimes v_{\tau_{0}}\right) / R_{2}$ is a generator of $L\left(\tau_{0}+\nu\right)$ we conclude that pr̃o $\left(\operatorname{pr}\left(e \otimes v_{\tau_{0}}\right)\right) / M\left(s_{\alpha} \cdot\left(\tau_{0}+\nu\right)\right) \in \mathrm{PO} / M\left(s_{\alpha} \cdot\left(\tau_{0}+\nu\right)\right)$ is also a generator of $L\left(\tau_{0}+\nu\right)$ and thus its preimage pro $\left(\operatorname{pr}\left(e \otimes v_{\tau_{0}}\right)\right)$ generates the indecomposable module $\mathrm{PO} \cong P\left(\tau_{0}+\nu\right)$. By Lemma 9 the element $z_{\tau_{0}}$ is not contained in the annihilator of $P\left(\tau_{0}+\nu\right)$, this means in particular $0 \neq z_{\tau_{0}} \operatorname{pro}\left(\operatorname{pr}\left(e \otimes v_{\tau_{0}}\right)\right)=\operatorname{prro}\left(z_{\tau_{0}} \operatorname{pr}\left(e \otimes v_{\tau_{0}}\right)\right)$ 
and we obtain $z_{\tau_{0}} \operatorname{pr}\left(e \otimes v_{\tau_{0}}\right) \neq 0$. Therefore, claim (**)' is proven and the proof of Theorem 1 is finished provided we know that Lemma 6, 7, 8 and 9 hold.

3.3. Proof of Lemmas 6, 7, 8 and 9. In the following let $e_{\nu}$ denote again the fixed extremal weight vector of the finite-dimensional irreducible $\mathfrak{g}$-module $E=$ $E(\nu)$. Set $v_{\tau} \in M(\tau)$ the canonical generator of the Verma module and let $E \otimes$ $\mathfrak{U}\left(\mathfrak{n}^{-}\right) \cong E \otimes M(\tau)$ for all $\tau \in \mathfrak{h}^{*}$. The category $\mathcal{O}$ is the category of all finitely generated $\mathfrak{g}$-modules, which are $\mathfrak{b}$-finite and semisimple over $\mathfrak{h}$ [BGG2]. For $\lambda \in \mathfrak{h}^{*}$ set $\mathcal{W}_{\lambda}:=\{w \in \mathcal{W} \mid \lambda-w \lambda \in P\}$ and $R_{\lambda}:=\left\{\beta \in R \mid\left\langle\lambda, \beta^{\vee}\right\rangle \in \mathbb{Z}\right\}$. The group $\mathcal{W}_{\lambda}$ is then the Weyl group to the root system $R_{\lambda}$ [Ja1, 1.3]. Denote by $P(\lambda)$ the projective cover in $\mathcal{O}$ of the simple module $L(\lambda)$.

Lemma 6. Let $\lambda \in \mathfrak{h}^{*}$ with $\mathcal{W}_{\lambda}=\left\langle s_{\alpha}\right\rangle$ and $M(\lambda)=L(\lambda)$ simple. If the short exact sequence $M\left(s_{\alpha} \cdot \lambda\right) \hookrightarrow N \rightarrow L(\lambda)$ is a nontrivial extension, then $N$ is isomorphic to $P(\lambda)$.

Proof. For $\lambda$ with $\mathcal{W}_{\lambda}=\left\langle s_{\alpha}\right\rangle$ there exists in $\mathcal{O}$ (up to isomorphism) a unique indecomposable projective module $P(\lambda)$, such that the short exact sequence

$$
M\left(s_{\alpha} \cdot \lambda\right) \hookrightarrow P(\lambda) \rightarrow L(\lambda)
$$

is a nontrivial extension [BGG2, Ch. 4]. We thus have a nontrivial element of $\operatorname{Ext}^{1}\left(L(\lambda), M\left(s_{\alpha} \cdot \lambda\right)\right)$. The assertion follows immediately if we know that $\operatorname{dim} \operatorname{Ext}^{1}\left(L(\lambda), M\left(s_{\alpha} \cdot \lambda\right)\right) \leq 1$. Indeed, construct for $M\left(s_{\alpha} \cdot \lambda\right) \hookrightarrow P(\lambda) \rightarrow L(\lambda)$ the long exact homology sequence [HS, Ch. 3, Thm. 5.3] ( $\operatorname{set} M=M\left(s_{\alpha} \cdot \lambda\right), P=$ $P(\lambda), L=L(\lambda)$, Ext $=$ Ext $_{\mathcal{O}}$ and let $\omega$ be the connecting homomorphism):

$$
\cdots \rightarrow \operatorname{Hom}(M, M) \stackrel{\omega}{\rightarrow} \operatorname{Ext}^{1}(L, M) \rightarrow \operatorname{Ext}^{1}(P, M) \rightarrow \operatorname{Ext}^{1}(M, M) \rightarrow \cdots
$$

and note that $\operatorname{Ext}^{1}(P, M)=0$ since $P=P(\lambda)$ is projective [HS, Chap. 3, Prop. 2.6]. By exactness we obtain $\omega$ surjective. Since now for any Verma module $\mathrm{M} \operatorname{dim} \operatorname{Hom}(M, M)=1$, we conclude that $\operatorname{dim} \operatorname{Ext}^{1}(L, M) \leq 1$.

Lemma 7. Let $\tau \in \mathfrak{h}^{*}$ with $\mathcal{W}_{\tau}=\left\langle s_{\alpha}\right\rangle$.

(a) If $\left\langle\tau+\rho, \alpha^{\vee}\right\rangle \geq 0$, then $\operatorname{pr}_{\chi(\tau+\nu)}(E \otimes M(\tau))$ is projective, this means that $\operatorname{pr}_{\chi(\tau+\nu)}(E \otimes M(\tau))$ is a projective object of the category $\mathcal{O}$.

(b) If $\left\langle\tau+\nu+\rho, \alpha^{\vee}\right\rangle \leq 0$, then $M(\tau+\nu)=L(\tau+\nu)$ is simple and not projective.

Proof. a) A Verma module $M(\lambda)$ is projective if $\left\langle\lambda+\rho, \beta^{\vee}\right\rangle \geq 0$ for all $\beta \in R_{\lambda} \cap R^{+}$ [Ja2, 4.8]. Since $\mathcal{W}_{\tau}=\left\langle s_{\alpha}\right\rangle$, we have $R_{\tau} \cap R^{+}=\{\alpha\}$ and hence $M(\tau)$ is projective. Then also $E \otimes M(\tau)$ is projective, because for $E$ with $\operatorname{dim} E<\infty$ the functor

$$
\begin{array}{clcc}
F_{E}: & \mathcal{O} & \longrightarrow & \mathcal{O} \\
& M & \mapsto & E \otimes M
\end{array}
$$

maps projective objects of $\mathcal{O}$ to projective objects of $\mathcal{O}$ [BG]. Its direct summand $\operatorname{pr}_{\chi(\tau+\nu)}(E \otimes M(\tau)) \subset E \otimes M(\tau)$ is then projective too.

b) A Verma module $M(\lambda)$ is simple if and only if $\left\langle\lambda+\rho, \beta^{\vee}\right\rangle \leq 0$ for all $\beta \in$ $R_{\lambda} \cap R^{+}$[Di, 7.6.24] or [Ja1, 1.8, 1.9]. Since $\mathcal{W}_{\tau+\nu}=\mathcal{W}_{\tau}=\left\langle s_{\alpha}\right\rangle$ we obtain $R_{\tau+\nu} \cap R^{+}=\{\alpha\}$ and the simplicity of $M(\tau+\nu)=L(\tau+\nu)$ follows. Since the short exact sequence $M\left(s_{\alpha} \cdot(\tau+\nu)\right) \hookrightarrow P(\tau+\nu) \rightarrow L(\tau+\nu)$ is a nontrivial extension [BGG2, Ch. 4], the module $L(\tau+\nu)$ cannot be projective. 
Lemma 8. Let $\tau \in \mathfrak{h}^{*}$ with $\mathcal{W}_{\tau}=\left\langle s_{\alpha}\right\rangle$ and $\mu_{0}:=\nu-\left\langle\tau+\nu+\rho, \alpha^{\vee}\right\rangle \alpha \in P(E)$ such that $\mu_{0}>\nu$. Then the module $\operatorname{pr}_{\chi(\tau+\nu)}(E \otimes M(\tau))$ admits a chain of submodules

$$
\operatorname{pr}_{\chi(\tau+\nu)}(E \otimes M(\tau))=R_{1} \supset R_{2} \supset 0
$$

such that $R_{2} \cong \bigoplus_{\operatorname{dim} E_{\mu_{0}}} M\left(s_{\alpha} \cdot(\tau+\nu)\right)$ and $R_{1} / R_{2} \cong M(\tau+\nu)=L(\tau+\nu)$ and such that $L(\tau+\nu)$ is generated by $\left(\operatorname{pr}_{\chi(\tau+\nu)}\left(e_{\nu} \otimes v_{\tau}\right)\right) / R_{2}$.

Proof. Let $\mu_{1}, \mu_{2}, \ldots, \mu_{m}$ be the weights of $E$ without multiplicities, numbered such that $\mu_{i}<\mu_{j} \Rightarrow i<j$. Set $\mu_{j_{0}}:=\nu, \mu_{j_{1}}:=\mu_{0}, d_{j}:=\operatorname{dim} E_{\mu_{j}}$ and $\operatorname{pr}:=$ $\operatorname{pr}_{\chi(\tau+\nu)}$. From Chapter 2 we already know that $E \otimes M(\tau)$ has a chain of submodules $E \otimes M(\tau)=M_{1} \supset \cdots \supset M_{m} \supset 0$ such that $M_{j} / M_{j+1} \cong \bigoplus_{d_{j}} M\left(\tau+\mu_{j}\right)$. It is then clear that $\operatorname{pr}(E \otimes M(\tau))=\operatorname{pr} M_{1} \supset \cdots \supset \operatorname{pr} M_{m} \supset 0$ is a chain of submodules such that the subquotients $\operatorname{pr} M_{j} / \operatorname{pr} M_{j+1}$ are isomorphic to $\bigoplus_{d_{j}} M\left(\tau+\mu_{j}\right)$, if $\tau+\mu_{j} \in \mathcal{W} \cdot(\tau+\nu)$, otherwise they are 0 . Since $\mu_{j}$ and $\nu$ are integral weights, we get $\mu_{j}=w \cdot(\tau+\nu)-\tau$ only for $w \in \mathcal{W}_{\tau}$. For $w=e$ this yields $\mu_{j_{0}}=\nu$, for $w=s_{\alpha}$ we obtain $\mu_{j_{1}}=\mu_{0}$ since $\tau+\mu_{0}=s_{\alpha} \cdot(\tau+\nu)$. By assumption we have $\mu_{j_{0}}=\nu<\mu_{0}=\mu_{j_{1}}$, and thus $j_{0}<j_{1}$. Therefore, if we omit in this chain trivial submodules we obtain a chain $\operatorname{pr}(E \otimes M(\tau))=\operatorname{pr} M_{j_{0}}=: R_{1} \supset$ $\operatorname{pr} M_{j_{1}}=: R_{2} \supset 0$ such that $R_{2} \cong \bigoplus_{d_{j_{1}}} M\left(\tau+\mu_{0}\right)=\bigoplus_{d_{j_{1}}} M\left(s_{\alpha} \cdot(\tau+\nu)\right)$ and $R_{1} / R_{2} \cong \bigoplus_{d_{j_{0}}} M(\tau+\nu) \cong M(\tau+\nu)$. By construction of the $M_{i}$ this module is generated by $\operatorname{pr}_{\chi(\tau+\nu)}\left(e_{\nu} \otimes v_{\tau}\right) / R_{2}$. Now $\mu_{0}>\nu$ forces $\left\langle\tau+\nu+\rho, \alpha^{\vee}\right\rangle<0$ and together with $\mathcal{W}_{\tau+\nu}=\mathcal{W}_{\tau}=\left\langle s_{\alpha}\right\rangle$ this implies $M(\tau+\nu)=L(\tau+\nu)$ (Lemma 7$)$.

For $\mu \in \mathfrak{h}^{*}$ define maps $H_{\mu}: \mathfrak{h}^{*} \times \mathfrak{h}^{*} \rightarrow k$ by $H_{\mu}(\lambda, \tau):=\left\langle\lambda-\tau-\mu, h_{\mu}\right\rangle \forall \lambda, \tau \in$ $\mathfrak{h}^{*}, h_{\mu} \in \mathfrak{h}$. For $\tau \in \mathfrak{h}^{*}$ set $p_{\tau}:=\prod_{\mu>\nu, \mu \in P(E)} \operatorname{sym} H_{\mu}(-, \tau)$ and $z_{\tau}:=\xi^{-1}\left(p_{\tau}\right) \in \mathfrak{Z}$ the preimage of $p_{\tau}$ under the Harish-Chandra isomorphism $\xi: \mathfrak{Z} \stackrel{\sim}{\longrightarrow} \mathcal{P}\left(\mathfrak{h}^{*}\right)^{\mathcal{W}}$.

Lemma 9. Let $\tau_{0} \in \mathfrak{h}^{*}$ with $\mathcal{W}_{\tau_{0}}=\left\langle s_{\alpha}\right\rangle$ and $-\left\langle\rho, \alpha^{\vee}\right\rangle \leq\left\langle\tau_{0}, \alpha^{\vee}\right\rangle \leq-\left\langle\nu+\rho, \alpha^{\vee}\right\rangle-1$. Assume the $h_{\mu} \in \mathfrak{h}$ to be chosen such that

(a) $\left\langle\alpha, h_{\mu_{0}}\right\rangle \neq 0$ for $\mu_{0}:=\nu-\left\langle\tau_{0}+\nu+\rho, \alpha^{\vee}\right\rangle \alpha$ and

(b) $H_{\mu}\left(w \cdot\left(\tau_{0}+\nu\right), \tau_{0}\right) \neq 0$ for $(w, \mu) \neq\left(s_{\alpha}, \mu_{0}\right), \forall w \in \mathcal{W}, \mu \in P(E)$ with $\mu>\nu$.

Then $z_{\tau_{0}} \notin \mathrm{Ann}_{\mathfrak{Z}} P\left(\tau_{0}+\nu\right)$.

Proof. We will first give an explicit description of the annihilator $\mathrm{Ann}_{\mathfrak{Z}} P\left(\tau_{0}+\nu\right)$ by a theorem of Soergel [So]. For this let $\lambda \in \mathfrak{h}^{*}$, such that for all $\beta \in R^{+} \cap R_{\lambda}$ we have $\left\langle\lambda+\rho, \beta^{\vee}\right\rangle \geq 0$. Denote by $w_{\lambda} \in \mathcal{W}_{\lambda}$ the longest element with respect to the Bruhat ordering. Then define for $\mu \in \mathfrak{h}^{*}$ the map

$$
\begin{aligned}
\mu^{+}: \mathcal{P}\left(\mathfrak{h}^{*}\right) & \longrightarrow \mathcal{P}\left(\mathfrak{h}^{*}\right) \\
p & \mapsto \mu^{+}(p)
\end{aligned}
$$

by $\left(\mu^{+}(p)\right)(\tau):=p(\tau+\mu)$ for all $\tau \in \mathfrak{h}^{*}$. Then [So, 2.2]:

$$
\xi^{-1}(p) \in \operatorname{Ann}_{\mathfrak{Z}} P\left(w_{\lambda} \cdot \lambda\right) \Longleftrightarrow \lambda^{+}(p) \in\left(\mathcal{P}^{+}\left(\mathfrak{h}^{*}\right)\right)^{\mathcal{W}_{\lambda}} \mathcal{P}\left(\mathfrak{h}^{*}\right)
$$

Here $\mathcal{P}^{+}\left(\mathfrak{h}^{*}\right)$ denotes the set of polynomial functions on $\mathfrak{h}^{*}$ without constant term. We want to describe the annihilator of $P\left(\tau_{0}+\nu\right)$ and choose for this $\lambda_{0}:=s_{\alpha}$. $\left(\tau_{0}+\nu\right)=\tau_{0}+\mu_{0}=\tau_{0}+\nu-\left\langle\tau_{0}+\nu+\rho, \alpha^{\vee}\right\rangle \alpha$. We then have $\mathcal{W}_{\lambda_{0}}=\mathcal{W}_{s_{\alpha} \cdot\left(\tau_{0}+\nu\right)}=$ $\mathcal{W}_{\tau_{0}+\nu}=\mathcal{W}_{\tau_{0}}=\left\langle s_{\alpha}\right\rangle$ and hence $R_{\lambda_{0}}=\{\alpha,-\alpha\}$. By assumption we know that $\left\langle\lambda_{0}+\rho, \alpha^{\vee}\right\rangle \geq 0$ and obtain for all $\beta \in R^{+} \cap R_{\lambda_{0}}$ that $\left\langle\lambda_{0}+\rho, \beta^{\vee}\right\rangle \geq 0$. Now $w_{\lambda_{0}}=s_{\alpha}$ is the longest element in $\mathcal{W}_{\lambda_{0}}$ and we may apply Soergel's theorem with 
$\lambda:=\lambda_{0}=s_{\alpha} \cdot\left(\tau_{0}+\nu\right)$. Assume we had $\xi^{-1}(p) \in \operatorname{Ann}_{\mathfrak{Z}} P\left(w_{\lambda_{0}} \cdot \lambda_{0}\right)=\operatorname{Ann}_{\mathfrak{Z}} P\left(\tau_{0}+\nu\right)$ for $p=\sum_{i=1}^{n} p_{i} q_{i}$ with $\left.p_{i} \in \mathcal{P}^{+}\left(\mathfrak{h}^{*}\right)\right)^{\mathcal{W}_{\lambda_{0}}}$ and $q_{i} \in \mathcal{P}\left(\mathfrak{h}^{*}\right)$. Then it follows for all $p_{i}$ that

$$
p_{i}\left(\lambda_{0}+\mu\right)=\left(\lambda_{0}^{+}\left(p_{i}\right)\right)(\mu)=\left(\lambda_{0}^{+}\left(p_{i}\right)\right)\left(s_{\alpha} \mu\right)=p_{i}\left(\lambda_{0}+s_{\alpha} \mu\right) \forall \mu \in \mathfrak{h}^{*} .
$$

For $\mu=\alpha$ we obtain $p_{i}\left(\lambda_{0}+\alpha\right)=p_{i}\left(\lambda_{0}-\alpha\right)$ and this forces the derivative of $p$ in direction of $\alpha$ to vanish at the point $\lambda_{0}$. Let us check this condition for $p_{\tau}$. By definition we have for all $\lambda \in \mathfrak{h}^{*}: p_{\tau}(\lambda)=\prod_{\mu>\nu, w \in \mathcal{W}} H_{\mu}(w \cdot \lambda, \tau)$. If we define $\bar{p}_{\tau}$ by $\bar{p}_{\tau} H_{\mu_{0}}(-, \tau)=p_{\tau}$, we obtain

$$
\bar{p}_{\tau}(\lambda):=\prod_{\substack{(w, \mu) \neq\left(e, \mu_{0}\right) \\ w \in \mathcal{W}, \mu>\nu}} H_{\mu}(w \cdot \lambda, \tau) .
$$

Let $p_{\tau}^{\prime}$ denote the derivative of $p_{\tau}$ in direction $\alpha$. By the product rule we have $p_{\tau}^{\prime}=\bar{p}_{\tau}^{\prime} H_{\mu_{0}}(-, \tau)+\bar{p}_{\tau} H_{\mu_{0}}^{\prime}(-, \tau)$.

Since $s_{\alpha} \cdot\left(\tau_{0}+\nu\right)=\tau_{0}+\mu_{0}$ it follows that $H_{\mu_{0}}\left(s_{\alpha} \cdot\left(\tau_{0}+\nu\right), \tau_{0}\right)=0$ and we get $p_{\tau_{0}}^{\prime}\left(s_{\alpha} \cdot\left(\tau_{0}+\nu\right)\right)=\bar{p}_{\tau_{0}}\left(s_{\alpha} \cdot\left(\tau_{0}+\nu\right)\right) H_{\mu_{0}}^{\prime}\left(s_{\alpha} \cdot\left(\tau_{0}+\nu\right), \tau_{0}\right)$. But now neither of these two factors is zero because

$$
\begin{aligned}
\bar{p}_{\tau_{0}}\left(s_{\alpha} \cdot\left(\tau_{0}+\nu\right)\right) & =\prod_{\substack{(w, \mu) \neq\left(e, \mu_{0}\right) \\
w \in \mathcal{W}, \mu>\nu}} H_{\mu}\left(w \cdot s_{\alpha} \cdot\left(\tau_{0}+\nu\right), \tau_{0}\right) \\
& =\prod_{\substack{w, \mu) \neq\left(s_{\alpha}, \mu_{0}\right) \\
w \in \mathcal{W}, \mu>\nu}} H_{\mu}\left(w \cdot\left(\tau_{0}+\nu\right), \tau_{0}\right)
\end{aligned}
$$

and by assumption (b) none of the factors $H_{\mu}\left(w \cdot\left(\tau_{0}+\nu\right), \tau_{0}\right)$ vanishes, hence $\bar{p}_{\tau_{0}}\left(s_{\alpha} \cdot\left(\tau_{0}+\nu\right)\right) \neq 0$. On the other hand, also $H_{\mu_{0}}^{\prime}\left(s_{\alpha} \cdot\left(\tau_{0}+\nu\right), \tau_{0}\right) \neq 0$ since by assumption (a) we have: $H_{\mu_{0}}^{\prime}\left(\lambda, \tau_{0}\right)=\left.\frac{\mathrm{d}}{\mathrm{d} t}\right|_{t=0}\left\langle\lambda+t \alpha-\tau_{0}-\mu_{0}, h_{\mu_{0}}\right\rangle=\left\langle\alpha, h_{\mu_{0}}\right\rangle \neq 0$.

Together this implies $p_{\tau_{0}}^{\prime}\left(s_{\alpha} \cdot\left(\tau_{0}+\nu\right)\right) \neq 0$ and therefore $\xi\left(p_{\tau_{0}}\right)=z_{\tau_{0}}$ cannot be contained in the annihilator of $P\left(\tau_{0}+\nu\right)$.

\section{The TRIAngle FUnCtion $\Delta$}

4.1. Preliminaries. Let $M$ be a representation of $\mathfrak{g}$ and $E$ a vector space. Then $E \otimes M$ is a representation of $\mathfrak{g}$ via $X(e \otimes m):=e \otimes X m$ for all $X \in \mathfrak{g}, e \in E$ and $m \in M$. If in addition $E$ is also a representation of $\mathfrak{g}$, then we obtain a second $\mathfrak{g}$-operation on $E \otimes M$ via $X(e \otimes m):=X e \otimes m+e \otimes X m$. To distinguish these two representations we denote the first one by $E \hat{\otimes} M$. Let now $E$ be a finite-dimensional representation of $\mathfrak{g}$ and $\nu \in P$ a weight of $E$.

Lemma 10. Let $\tau \in \mathfrak{h}^{*}$ such that $\chi(\tau+\nu) \neq \chi(\tau+\mu)$ for all $\mu \in P(E)$ with $\mu \neq \nu$. Then there exists a unique natural isomorphism

$$
\text { can : } E_{\nu} \hat{\otimes} M(\tau+\nu) \stackrel{\sim}{\longrightarrow} \operatorname{pr}_{\chi(\tau+\nu)}(E \otimes M(\tau)),
$$

such that $\operatorname{can}\left(e \hat{\otimes} v_{\tau+\nu}\right)=\operatorname{pr}_{\chi(\tau+\nu)}\left(e \otimes v_{\tau}\right)$ for all $e \in E_{\nu}$.

Remark. For a generic weight, i.e. a weight $\tau \in \mathfrak{h}^{*}$ such that $\left\langle\tau, \alpha^{\vee}\right\rangle \notin \mathbb{Z}$ for all $\alpha \in R$, the central characters $\chi(\tau+\mu)$ for $\mu \in P(E)$ are pairwise distinct. In particular, in this case the condition of the lemma is always satisfied. 
Proof. By the so-called tensor identity we have a canonical isomorphism

$$
\mathfrak{U} \otimes_{\mathfrak{U}(\mathfrak{b})}\left(E \otimes k_{\tau}\right) \stackrel{\sim}{\longrightarrow} E \otimes\left(\mathfrak{U} \otimes_{\mathfrak{U}(\mathfrak{b})} k_{\tau}\right)
$$

such that $u \otimes(e \otimes a) \mapsto u(e \otimes(1 \otimes a))$. Call the left hand side $F$, the right hand side is $E \otimes M(\tau)$. Denote by $\mu_{1}, \ldots, \mu_{m}$ the weights of $E$. The filtration $E \otimes M(\tau)=M_{1} \supset \cdots \supset M_{m} \supset 0$, where the subquotients are isomorphic to direct sums of Verma modules (see Chapter 2), induces a filtration of $F: \mathfrak{U} \otimes \mathfrak{U}(\mathfrak{b})\left(E \otimes k_{\tau}\right)=$ $F=F_{1} \supset F_{2} \supset \cdots \supset F_{m} \supset 0$ such that

$$
F_{j} / F_{j+1} \cong \mathfrak{U} \otimes_{\mathfrak{U}(\mathfrak{b})}\left(E_{\mu_{j}} \otimes k_{\tau}\right) .
$$

Now here the right hand side is canonically isomorphic to $E_{\mu_{j}} \hat{\otimes} M\left(\tau+\mu_{j}\right)$ (by mapping $\left.e \otimes u v_{\tau+\mu_{j}} \mapsto u \otimes(e \otimes 1)\right)$ and in particular, we have that $\chi\left(\tau+\mu_{j}\right)\left(F_{j} / F_{j+1}\right)=$ 0 .

Let now $\nu=\mu_{i}$ for a fixed $i$. By the condition on $\tau$ we know that $\chi(\tau+\nu) \neq$ $\chi\left(\tau+\mu_{j}\right)$ for all $j \neq i, j \in\{1, \ldots, m\}$ and hence $\operatorname{pr}_{\chi(\tau+\nu)}\left(F_{j} / F_{j+1}\right)=0$ for all $j \neq i$. Thus we get $\operatorname{pr}_{\chi(\tau+\nu)} F=\operatorname{pr}_{\chi(\tau+\nu)} F_{1}=\ldots=\operatorname{pr}_{\chi(\tau+\nu)} F_{i}$ and also $\operatorname{pr}_{\chi(\tau+\nu)} F_{i+1}=\ldots=\operatorname{pr}_{\chi(\tau+\nu)} F_{m}=0$. We conclude that $\operatorname{pr}_{\chi(\tau+\nu)} F \subset F_{i}$ and that $F_{i+1} \subset \operatorname{ker}\left(\operatorname{pr}_{\chi(\tau+\nu)}: F_{i} \rightarrow \operatorname{pr}_{\chi(\tau+\nu)} F\right)$. Since $\operatorname{pr}_{\chi(\tau+\nu)}\left(F_{i} / F_{i+1}\right)=F_{i} / F_{i+1}$, we even know that $F_{i+1}$ is equal to this kernel. This now induces a natural isomorphism

$$
F_{i} / F_{i+1} \stackrel{\sim}{\longrightarrow} \operatorname{pr}_{\chi(\tau+\nu)} F
$$

such that

$$
\begin{array}{clc}
E_{\nu} \hat{\otimes} M(\tau+\nu) & \stackrel{\sim}{\longrightarrow} & \operatorname{pr}_{\chi(\tau+\nu)} F \\
e \hat{\otimes}\left(u v_{\tau+\nu}\right) & \longmapsto & \operatorname{pr}_{\chi(\tau+\nu)}(u \otimes(e \otimes 1)) .
\end{array}
$$

We apply the tensor identity $F \cong E \otimes M(\tau)$ and the lemma follows.

Let us recall the theorem of Bernstein and Gelfand [BG] for projective functors. For this denote by $\mathcal{M}$ the category of all $\mathfrak{Z}$-finite $\mathfrak{g}$-modules and for $\chi \in \operatorname{Max} \mathfrak{Z}$ let

$$
\begin{aligned}
\mathcal{M}(\chi) & :=\{M \in \mathcal{M} \mid \chi M=0\}, \\
\mathcal{M}^{\infty}(\chi) & :=\left\{M \in \mathcal{M} \mid \text { for all } m \in M \text { exists } n \in \mathbb{N} \text { such that } \chi^{n} m=0\right\} .
\end{aligned}
$$

A projective $\chi$-functor is then a functor $F: \mathcal{M}(\chi) \rightarrow \mathcal{M}$, which is isomorphic to a direct summand of a functor $E \otimes$ for a finite-dimensional representation $E$. In particular, the restriction of the translation functor $T_{\tau}^{\tau+\nu}: \mathcal{M}^{\infty}(\chi(\tau)) \rightarrow$ $\mathcal{M}^{\infty}(\chi(\tau+\nu))$ to the subcategory $\mathcal{M}(\chi(\tau))$ is a projective $\chi(\tau)$-functor. For two

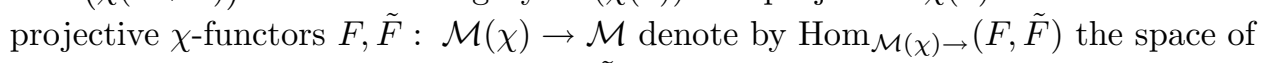
all natural transformations from $F$ to $\tilde{F}$.

Theorem $([\mathrm{BG}, 3.5])$. Let $F, \tilde{F}: \mathcal{M}(\chi) \rightarrow \mathcal{M}$ be projective $\chi$-functors and let $\tau \in \mathfrak{h}^{*}$ such that $\chi(\tau)=\chi$ and $M(\tau)$ is projective. Then the obvious map

$$
\operatorname{Hom}_{\mathcal{M}(\chi(\tau)) \rightarrow}(F, \tilde{F}) \stackrel{\sim}{\longrightarrow} \operatorname{Hom}_{\mathfrak{g}}(F M(\tau), \tilde{F} M(\tau))
$$

is an isomorphism.

Remark. (i) The Verma module $M(\tau)$ is projective if and only if $\left\langle\tau+\rho, \alpha^{\vee}\right\rangle \notin$ $\{-1,-2, \ldots\}$ for all $\alpha \in R^{+}$. In particular, for a generic weight $\tau$ the Verma module $M(\tau)$ is always projective.

(ii) Note that in $[\mathrm{BG}]$ the Verma module with highest weight $\tau$ is denoted by $M_{\tau+\rho}$. Accordingly, the theorem there is formulated for all $\tau$ with $\left\langle\tau, \alpha^{\vee}\right\rangle \notin$ $\{-1,-2, \ldots\}$ for all $\alpha \in R^{+}$. 
4.2. Definition of $\Delta$. For $\nu \in P$ let $E(\nu)$ be the finite-dimensional irreducible $\mathfrak{g}$-module with extremal weight $\nu$ and let $x \in \mathcal{W}$. For a weight $\tau \in \mathfrak{h}^{*}$ with $\chi(\tau+\nu) \neq \chi(\tau+\mu)$ for all $\mu \in P(E)$ with $\mu \neq \nu$, Lemma 10 yields a canonical isomorphism

$$
E(\nu)_{x \nu} \hat{\otimes} M(x \cdot(\tau+\nu)) \stackrel{\sim}{\longrightarrow} \operatorname{pr}_{\chi(\tau+\nu)}(E(\nu) \otimes M(x \cdot \tau))=T_{\tau}^{\tau+\nu} M(x \cdot \tau) .
$$

Let now $\nu, \mu \in P$ be integral weights. We set $E^{\prime}:=E(\nu), E^{\prime \prime}:=E(\mu)$ and $E:=E(\nu+\mu)$. For generic $\tau$ consider the following sequence of isomorphisms:

$$
\begin{aligned}
& \operatorname{Hom}_{\mathcal{M}(\chi(\tau)) \rightarrow}\left(T_{\tau+\nu}^{\tau+\nu+\mu} \circ T_{\tau}^{\tau+\nu}, T_{\tau}^{\tau+\nu+\mu}\right) \\
& \stackrel{\sim}{\longrightarrow} \operatorname{Hom}_{\mathfrak{g}}\left(T_{\tau+\nu}^{\tau+\nu+\mu} T_{\tau}^{\tau+\nu} M(x \cdot \tau), T_{\tau}^{\tau+\nu+\mu} M(x \cdot \tau)\right) \\
& \stackrel{\sim}{\longrightarrow} \operatorname{Hom}_{\mathfrak{g}}\left(E_{x \mu}^{\prime \prime} \hat{\otimes} E_{x \nu}^{\prime} \hat{\otimes} M(x \cdot(\tau+\nu+\mu)), E_{x(\mu+\nu)} \hat{\otimes} M(x \cdot(\tau+\nu+\mu))\right) \\
& \stackrel{\sim}{\longrightarrow} \operatorname{Hom}_{k}\left(E_{x \mu}^{\prime \prime} \otimes E_{x \nu}^{\prime}, E_{x(\mu+\nu)}\right) \\
& \stackrel{\sim}{\longrightarrow} E_{x \mu}^{\prime \prime *} \otimes E_{x \nu}^{\prime *} \otimes E_{x(\mu+\nu)} .
\end{aligned}
$$

Here, we obtain the first isomorphism by the Theorem of Bernstein-Gelfand, the second is due to Lemma 10, the others are obvious. We call this map nat $(\mu, \nu ; x)(\tau)$ and define for generic $\tau$ and for the triangle

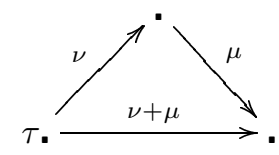

the value of the triangle function $\Delta$ by

$$
\Delta(\mu, \nu ; x)(\tau):=\operatorname{det}\left(x^{-1} \circ \operatorname{nat}(\mu, \nu ; x)(\tau) \circ(\operatorname{nat}(\mu, \nu ; e)(\tau))^{-1}\right) .
$$

We have yet to explain the map

$$
x^{-1}: E(\mu)_{x \mu}^{*} \otimes E(\nu)_{x \nu}^{*} \otimes E(\mu+\nu)_{x(\nu+\mu)} \rightarrow E(\mu)_{\mu}^{*} \otimes E(\nu)_{\nu}^{*} \otimes E(\mu+\nu)_{\nu+\mu} .
$$

For this let $G$ be a simply connected algebraic group with Lie algebra $\mathfrak{g}$ and $T \subset G$ a maximal torus with Lie algebra $\mathfrak{h}$. Each finite-dimensional representation $E$ of $\mathfrak{g}$ is in a natural way a representation of $G$. The operation of $N_{G}(T)$, the normalizer of $T$ in $G$, on $E$ stabilizes $E_{0}$ and factors over an operation of $\mathcal{W}=$ $N_{G}(T) / T$. The map $x^{-1}$ is given by this operation of $\mathcal{W}$ on the zero weight space $\left(E(\mu)^{*} \otimes E(\nu)^{*} \otimes E(\nu+\mu)\right)_{0}$.

Now take for $\tau$ not only any, but rather the generic weight: For this denote by $S:=S_{k}(\mathfrak{h})$ the symmetric algebra of $\mathfrak{h}$ and let $K:=$ Quot $(S)$ be its quotient field. We then have a $k$-linear map $\mathfrak{h} \hookrightarrow S \hookrightarrow K$ and obtain thus a $K$-linear map $\tau: K \otimes_{k} \mathfrak{h} \longrightarrow K$ such that the following diagram commutes

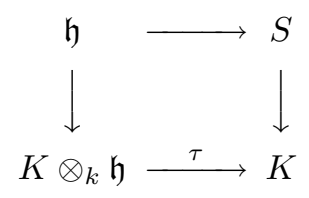

With this $\tau$ (= tautologous) we then obtain $\Delta(\mu, \nu ; x)(\tau) \in K^{\times}$. These are the triangle functions. 
4.3. Uncanonical definition of $\Delta$. We defined the triangle functions by a series of canonical isomorphisms. For our purposes it is sometimes more convenient to realize the triangle functions in the following - uncanonical-way: Let $\nu \in P, x \in$ $\mathcal{W}$ and choose a fixed extremal weight vector $0 \neq e_{\nu} \in E(\nu)_{\nu}$. Since extremal weight spaces are one dimensional, this choice is unique up to non-zero scalar. By Lemma 10 we obtain for generic $\tau \in \mathfrak{h}^{*}$ a-no longer canonical-isomorphism

$$
\begin{aligned}
& F_{x \nu}(x \cdot \tau): M(x \cdot(\tau+\nu)) \stackrel{\sim}{\longrightarrow} T_{\tau}^{\tau+\nu} M(x \cdot \tau)
\end{aligned}
$$

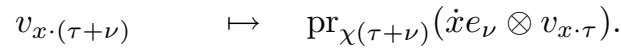

Here $\dot{x} \in G$ denotes a pre-image of $x \in \mathcal{W} \cong N_{G} T / T$. We have $\dot{x} e_{\nu} \in E(\nu)_{x \nu}$. Let now $\mu \in P$ be another weight. Choose $\tilde{e}_{\mu} \in E(\mu)_{\mu}$ and $\bar{e}_{\mu+\nu} \in E(\mu+\nu)_{\mu+\nu}$, both non-zero, and consider for generic $\tau$ the following sequence of isomorphisms:

$$
\begin{aligned}
\operatorname{Hom}_{\mathcal{M}(\chi(\tau))} & \rightarrow\left(T_{\tau+\nu}^{\tau+\nu+\mu} \circ T_{\tau}^{\tau+\nu}, T_{\tau}^{\tau+\nu+\mu}\right) \\
& \stackrel{\sim}{\longrightarrow} \operatorname{Hom}_{\mathfrak{g}}\left(T_{\tau+\nu}^{\tau+\nu+\mu} T_{\tau}^{\tau+\nu} M(x \cdot \tau), T_{\tau}^{\tau+\nu+\mu} M(x \cdot \tau)\right) \\
& \stackrel{\sim}{\longrightarrow} \operatorname{Hom}_{\mathfrak{g}}(M(x \cdot(\tau+\nu+\mu)), M(x \cdot(\tau+\nu+\mu))) \\
& \stackrel{\sim}{\longrightarrow} k .
\end{aligned}
$$

Denote this map by $\operatorname{Nat}(\mu, \nu ; x)(\tau)$. Here again the first isomorphism is clear by the Theorem of Bernstein-Gelfand, the second is due to the maps $F_{x(\nu+\mu)}(x \cdot \tau)$ and $F_{x \mu}(x \cdot(\tau+\mu)) \circ T_{\tau+\nu}^{\tau+\nu+\mu} F_{x \nu}(x \cdot \tau)$. We then obtain

$$
\Delta(\mu, \nu ; x)(\tau)=\operatorname{Nat}(\mu, \nu ; x)(\tau) \circ(\operatorname{Nat}(\mu, \nu ; e)(\tau))^{-1}(1) .
$$

It follows that this characterization of $\Delta$ is independent of the choice of the weight vectors $e_{\nu}, \tilde{e}_{\mu}$ and $\bar{e}_{\mu+\nu}$, and also independent of the choice of the pre-image $\dot{x}$ of $x$.

\section{Bernstein's Relative trace AND a SPECial CASE of $\Delta$}

5.1. The relative trace. Let us recall the definition of the relative $\operatorname{trace} \operatorname{tr}_{E}$ defined by Bernstein in [Be]. Let $\mathfrak{g}$-mod be the category of all $\mathfrak{g}$-modules and let $E$ be a finite-dimensional $\mathfrak{g}$-module. Denote by $F_{E}: \mathfrak{g}$-mod $\rightarrow \mathfrak{g}$-mod the functor defined by $F_{E}(M):=E \otimes M$. The relative trace $\operatorname{tr}_{E}: \operatorname{End}_{\mathfrak{g}-\bmod }\left(F_{E}\right) \rightarrow \operatorname{End}_{\mathfrak{g}-\bmod }(\mathrm{Id})$ is then a morphism from the endomorphisms of the functor $F_{E}$ to the endomorphisms of the identity functor on $\mathfrak{g}$-mod, defined by

$$
\begin{array}{ccc}
\operatorname{tr}_{E}^{M}: \operatorname{End}_{\mathfrak{g}}(E \otimes M) & \rightarrow \operatorname{End}_{\mathfrak{g} M} M \\
a & \mapsto \operatorname{tr}_{E}^{M}(a)
\end{array}
$$

where

$$
\operatorname{tr}_{E}^{M}(a): M \stackrel{\mathrm{i}}{\longrightarrow} E^{*} \otimes E \otimes M \stackrel{\mathrm{id} \otimes a}{\longrightarrow} E^{*} \otimes E \otimes M \stackrel{\operatorname{cont} \otimes \mathrm{id}}{\longrightarrow} M .
$$

Here, i is the map $M \stackrel{\mathrm{j}}{\hookrightarrow} \operatorname{End}_{\mathfrak{g}}(E) \otimes M \stackrel{\mathrm{c}}{\rightarrow} E^{*} \otimes E \otimes M$ with $\mathrm{j}(m):=\operatorname{id}_{E} \otimes m$ and $\mathrm{c}$ the canonical isomorphism $\operatorname{End}_{\mathfrak{g}}(E) \cong E^{*} \otimes E$. The map cont : $E^{*} \otimes E \rightarrow k$ denotes the evaluation map.

Bernstein has calculated an explicit formula for the relative trace, by considering it as a map from $\mathcal{P}\left(\mathfrak{h}^{*}\right)^{\mathcal{W}}$. to itself in the following way: First, we identify $\operatorname{End}_{\mathfrak{g} \text {-mod }}(\mathrm{Id}) \cong \mathfrak{Z}$ with the center of the enveloping algebra $\mathfrak{U}$, then we make use of the natural morphism $\mathfrak{Z} \rightarrow \operatorname{End}_{\mathfrak{g}-\bmod }\left(F_{E}\right)$ and composing this with the trace map we obtain $\operatorname{tr}_{E}: \mathfrak{Z} \rightarrow \mathfrak{Z}$. By means of the Harish-Chandra isomorphism 
$\xi: \mathfrak{Z} \stackrel{\sim}{\longrightarrow} \mathcal{P}\left(\mathfrak{h}^{*}\right)^{\mathcal{W}}$. (normalized by $z-\xi(z) \in \mathfrak{U} \mathfrak{n}$ ) we may then regard $\operatorname{tr}_{E}$ as an endomorphism of $\mathcal{P}\left(\mathfrak{h}^{*}\right)^{\mathcal{W}}$.

Define now on $\mathcal{P}\left(\mathfrak{h}^{*}\right)$ a convolution $f \mapsto P(E) * f$ by

$$
(P(E) * f)(\lambda):=\sum_{\mu \in P(E)} f(\lambda+\mu),
$$

where the sum is taken over all weights $\mu \in P(E)$ with their multiplicities. Set $\Lambda(\lambda):=\prod_{\alpha \in R^{+}}\left\langle\lambda+\rho, \alpha^{\vee}\right\rangle$. Then we have

Theorem $([\mathrm{Be}]) \cdot \operatorname{tr}_{E}(f)=\Lambda^{-1}(P(E) * \Lambda f)$ for all $f \in \mathcal{P}\left(\mathfrak{h}^{*}\right)^{\mathcal{W}}$.

If we choose now for $M$ the Verma module $M(\lambda)$ we can associate to each endomorphism $f \in \operatorname{End}_{\mathfrak{g}}(E \otimes M(\lambda))$ an element $\operatorname{tr}_{E}^{M(\lambda)}(f)$ of $\operatorname{End}_{\mathfrak{g}}(M(\lambda)) \cong k$. As an endomorphism of $M(\lambda)$ this element operates on $M(\lambda)$ by multiplication with the scalar $\left(\operatorname{tr}_{E}^{M(\lambda)}(f)\right)(\lambda)$ and we obtain by Bernstein's Theorem for all $\lambda \in \mathfrak{h}^{*}$ and for all $w \in \mathcal{W}$

$$
\begin{aligned}
\left(\operatorname{tr}_{E}^{M(w \cdot \lambda)}(f)\right)(\lambda) & =\left(\Lambda^{-1}(P(E) * \Lambda f)\right)(\lambda) \\
& =(\Lambda(\lambda))^{-1} \sum_{\mu \in P(E)} \Lambda(\lambda+\mu) f(\lambda+\mu) .
\end{aligned}
$$

5.2. The special case $\Delta\left(-\nu, \nu ; w_{0}\right)$. Let now $E=E(\nu), w_{0} \in \mathcal{W}$ the longest element and $\operatorname{pr}_{\chi(\tau+\nu)} \in \operatorname{End}_{\mathfrak{g}}\left(E(\nu) \otimes M\left(w_{0} \cdot \tau\right)\right)$ the projection on the central character $\chi(\tau+\nu)$. Then $\operatorname{tr}_{E(\nu)}^{M\left(w_{0} \cdot \tau\right)}\left(\operatorname{pr}_{\chi(\tau+\nu)}\right)$ is an element in $\operatorname{End}_{\mathfrak{g}}\left(M\left(w_{0} \cdot \tau\right)\right) \cong k$ and we have

Theorem 2. Let $\nu \in P^{+}$be a dominant weight and $\tau \in \mathfrak{h}^{*}$ generic. Then

$$
\Delta\left(-\nu, \nu ; w_{0}\right)(\tau)=\operatorname{tr}_{E(\nu)}^{M\left(w_{0} \cdot \tau\right)}\left(\operatorname{pr}_{\chi(\tau+\nu)}\right) .
$$

Proof. Postponed to 5.3.

Regarding $\Delta\left(-\nu, \nu ; w_{0}\right)$ as a polynomial function on $\mathfrak{h}^{*}$ we obtain for this special case an explicit formula:

Corollary. Let $\nu \in P^{+}$be a dominant weight and $\tau \in \mathfrak{h}^{*}$ generic. Then

$$
\Delta\left(-\nu, \nu ; w_{0}\right)(\tau)=\prod_{\alpha \in R^{+}} \frac{\left\langle\tau+\nu+\rho, \alpha^{\vee}\right\rangle}{\left\langle\tau+\rho, \alpha^{\vee}\right\rangle} .
$$

Proof. Under the morphism $\mathcal{P}\left(\mathfrak{h}^{*}\right)^{\mathcal{W}} \cong \mathfrak{Z} \rightarrow \operatorname{End}_{\mathfrak{g} \text {-mod }}\left(F_{E}\right)$ the projection $\operatorname{pr}_{\chi(\tau+\nu)}$ is the image of a polynomial function, which takes value 1 at all weights $\lambda \in \mathcal{W} \cdot(\tau+\nu)$ and vanishes at all other weights $\tau+\mu$ with $\mu \in P(E)$. Call this polynomial function pr. By Bernstein's formula for the relative trace we then obtain

$$
\left(\operatorname{tr}_{E(\nu)}^{M\left(w_{0} \cdot \tau\right)}(\overline{\mathrm{pr}})\right)(\tau)=(\Lambda(\tau))^{-1} \sum_{\mu \in P(E(\nu))} \Lambda(\tau+\mu) \overline{\operatorname{pr}}(\tau+\mu)
$$

and by definition of $\overline{\mathrm{pr}}$ the value of $\overline{\operatorname{pr}}(\tau+\mu)$ for $\mu \in P(E)$ does not vanish if and only if there is a $w \in \mathcal{W}$ such that $w \cdot(\tau+\mu)=(\tau+\nu)$. Since $\tau$ is generic, this is only possible for $w=e$ and hence $\mu=\nu$. In this case we have $\overline{\operatorname{pr}}(\tau+\nu)=1$ and 
since furthermore $\operatorname{dim} E(\nu)_{\nu}=1$, we get $\sum_{\mu \in P(E(\nu))} \Lambda(\tau+\mu) \overline{\operatorname{pr}}(\tau+\mu)=\Lambda(\tau+\nu)$. The claim now follows by Theorem 2 and the equation

$$
\left(\operatorname{tr}_{E(\nu)}^{M\left(w_{0} \cdot \tau\right)}(\overline{\operatorname{pr}})\right)(\tau)=\frac{\Lambda(\tau+\nu)}{\Lambda(\tau)}=\prod_{\alpha \in R^{+}} \frac{\left\langle\tau+\nu+\rho, \alpha^{\vee}\right\rangle}{\left\langle\tau+\rho, \alpha^{\vee}\right\rangle}
$$

5.3. Proof of Theorem 2. First we make some more general preliminary remarks.

5.3.1. The adjunctions $\left(T_{\tau}^{\tau+\nu}, T_{\tau+\nu}^{\tau}\right)$ and $\left(T_{\tau+\nu}^{\tau}, T_{\tau}^{\tau+\nu}\right)$. Let $\mathcal{A}$ and $\mathcal{B}$ be categories and $F: \mathcal{A} \rightarrow \mathcal{B}, G: \mathcal{B} \rightarrow \mathcal{A}$ two functors. Then an adjunction $(F, G)$ of $F$ and $G$ is a family of isomorphisms

$$
(F, G)_{M, N}:=(F, G): \operatorname{Hom}_{\mathcal{B}}(F M, N) \stackrel{\sim}{\longrightarrow} \operatorname{Hom}_{\mathcal{A}}(M, G N),
$$

which is natural in $M$ and $N(M \in \mathcal{A}, N \in \mathcal{B})$.

For example, for $E$ a finite-dimensional $\mathfrak{g}$-module we obtain an adjunction $\left(F_{E}, F_{E^{*}}\right)$ of $F_{E}: \mathfrak{g}-\bmod \rightarrow \mathfrak{g}-\bmod$ and $F_{E^{*}}: \mathfrak{g}-\bmod \rightarrow \mathfrak{g}$-mod as the composition

$$
\operatorname{Hom}_{\mathfrak{g}}(E \otimes M, N) \rightarrow \operatorname{Hom}_{\mathfrak{g}}\left(E^{*} \otimes E \otimes M, E^{*} \otimes N\right) \rightarrow \operatorname{Hom}_{\mathfrak{g}}\left(M, E^{*} \otimes N\right) .
$$

Here, the first map is given by $f \mapsto \mathrm{id}_{E^{*}} \otimes f$, the second by $g \mapsto g \circ \mathrm{i}$. Interchanging $E$ and $E^{*}$ we obtain in the same way an adjunction $\left(F_{E^{*}}, F_{E}\right)$. Its inverse is the composition

$$
\operatorname{Hom}_{\mathfrak{g}}(M, E \otimes N) \rightarrow \operatorname{Hom}_{\mathfrak{g}}\left(E^{*} \otimes M, E^{*} \otimes E \otimes N\right) \rightarrow \operatorname{Hom}_{\mathfrak{g}}\left(E^{*} \otimes M, N\right),
$$

where the first map is again given by $f \mapsto \operatorname{id}_{E^{*}} \otimes f$ and the second by $g \mapsto$ $\left(\right.$ cont $\left.\otimes \operatorname{id}_{N}\right) \circ g$. Let now $\nu \in P$ be an integral weight. Each identification $\varphi: E(\nu)^{*} \stackrel{\sim}{\longrightarrow} E(-\nu)$ then defines adjunctions $\left(F_{E(\nu)}, F_{E(-\nu)}\right)$ and $\left(F_{E(-\nu)}, F_{E(\nu)}\right)$. More precisely, we have

$$
\begin{aligned}
& \left(F_{E(\nu)}, F_{E(-\nu)}\right): \quad \operatorname{Hom}_{\mathfrak{g}}(E(\nu) \otimes M, N) \stackrel{\sim}{\longrightarrow} \operatorname{Hom}_{\mathfrak{g}}(M, E(-\nu) \otimes N) \\
& f \quad \mapsto \quad(\varphi \otimes f) \circ \mathrm{i}
\end{aligned}
$$

and

$$
\begin{aligned}
& \left(F_{E(-\nu)}, F_{E(\nu)}\right)^{-1}: \quad \operatorname{Hom}_{\mathfrak{g}}(M, E(\nu) \otimes N) \stackrel{\sim}{\longrightarrow} \operatorname{Hom}_{\mathfrak{g}}(E(-\nu) \otimes M, N) \\
& g \quad \mapsto \quad\left(\operatorname{cont} \otimes \operatorname{id}_{N}\right) \circ\left(\varphi^{-1} \otimes g\right) .
\end{aligned}
$$

Let now $\mathrm{i}_{\chi}: \mathcal{M}^{\infty}(\chi) \hookrightarrow \mathcal{M}$ denote the embedding functor. We then have in a natural way adjunctions $\left(\mathrm{i}_{\chi}, \operatorname{pr}_{\chi}\right)$ and $\left(\operatorname{pr}_{\chi}, \mathrm{i}_{\chi}\right)$. Since for the translation functor $T_{\tau}^{\tau+\nu}=\operatorname{pr}_{\chi(\tau+\nu)} \circ F_{E(\nu)} \circ \mathrm{i}_{\chi(\tau)}$, we thus obtain also adjunctions $\left(T_{\tau}^{\tau+\nu}, T_{\tau+\nu}^{\tau}\right)$ and $\left(T_{\tau+\nu}^{\tau}, T_{\tau}^{\tau+\nu}\right)$.

5.3.2. The natural transformations $\operatorname{adj}^{1}$ and $\operatorname{adj}^{2}$. Let $M \in \mathcal{M}^{\infty}(\chi(\tau))$ and consider Id $\in \operatorname{Hom}_{\mathfrak{g}}\left(T_{\tau}^{\tau+\nu} M, T_{\tau}^{\tau+\nu} M\right)$. By means of the two adjunctions $\left(T_{\tau}^{\tau+\nu}, T_{\tau+\nu}^{\tau}\right)$ and $\left(T_{\tau+\nu}^{\tau}, T_{\tau}^{\tau+\nu}\right)^{-1}$ we get two maps as the images of Id:

$$
\operatorname{adj}_{M}^{1} \in \operatorname{Hom}_{\mathfrak{g}}\left(M, T_{\tau+\nu}^{\tau} T_{\tau}^{\tau+\nu} M\right) \text { and } \operatorname{adj}_{M}^{2} \in \operatorname{Hom}_{\mathfrak{g}}\left(T_{\tau+\nu}^{\tau} T_{\tau}^{\tau+\nu} M, M\right)
$$

and one checks that we obtain in this way natural transformations adj ${ }^{1} \in$

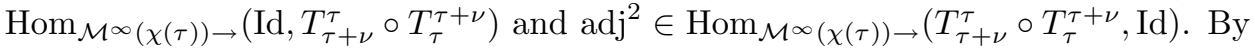
composing these two maps, we get a canonical endomorphism of $M$ :

$$
M \stackrel{\operatorname{adj}_{M}^{1}}{\longrightarrow} T_{\tau+\nu}^{\tau} T_{\tau}^{\tau+\nu} M \stackrel{\operatorname{adj}_{M}^{2}}{\longrightarrow} M .
$$


We want to describe this endomorphism in more detail. By definition, the adjunction $\left(T_{\tau}^{\tau+\nu}, T_{\tau+\nu}^{\tau}\right)$ is just the composition of adjunctions $\left(\mathrm{i}_{\chi(\tau)}, \mathrm{pr}_{\chi(\tau)}\right) \circ\left(F_{E(\nu)}\right.$, $\left.F_{E(-\nu)}\right) \circ\left(\operatorname{pr}_{\chi(\tau+\nu)}, \mathrm{i}_{\chi(\tau+\nu)}\right)$ and one checks easily that the image of

$$
\operatorname{Id} \in \operatorname{Hom}_{\mathfrak{g}}\left(T_{\tau}^{\tau+\nu} M, T_{\tau}^{\tau+\nu} M\right)
$$

under $\left(\operatorname{pr}_{\chi(\tau+\nu)}, \mathrm{i}_{\chi(\tau+\nu)}\right)$ is precisely the projection $\operatorname{pr}_{\chi(\tau+\nu)}$. Thus, the image of Id under the adjunction $\left(T_{\tau}^{\tau+\nu}, T_{\tau+\nu}^{\tau}\right)$ can be described by the composition

$$
\operatorname{adj}_{M}^{1}: M \stackrel{\mathrm{i}}{\longrightarrow} E^{*} \otimes E \otimes M \stackrel{\varphi \otimes \mathrm{pr}}{\longrightarrow} T_{\tau+\nu}^{\tau} T_{\tau}^{\tau+\nu} M .
$$

Here, we put $E=E(\nu)$ and $\mathrm{pr}=\operatorname{pr}_{\chi(\tau+\nu)}$. Analogously, we obtain for $\operatorname{adj}_{M}^{2}$ the composition

$$
\operatorname{adj}_{M}^{2}: T_{\tau+\nu}^{\tau} T_{\tau}^{\tau+\nu} M \stackrel{\varphi^{-1} \otimes \mathrm{pr}}{\longrightarrow} E^{*} \otimes E \otimes M \stackrel{\operatorname{cont} \otimes \mathrm{id}_{M}}{\longrightarrow} M
$$

and taken together we have the following commutative diagram:

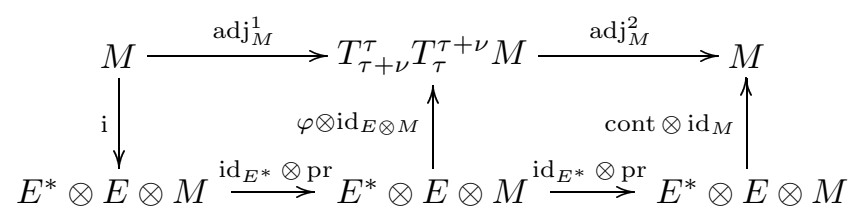

We thus obtain for $\operatorname{adj}_{M}^{2} \circ \operatorname{adj}_{M}^{1}$

$$
M \stackrel{\mathrm{i}}{\longrightarrow} E^{*} \otimes E \otimes M \stackrel{\operatorname{id}_{E^{*}} \otimes \operatorname{pr}_{\chi(\tau+\nu)}}{\longrightarrow} E^{*} \otimes E \otimes M \stackrel{\operatorname{cont} \otimes \operatorname{id}_{M}}{\longrightarrow} M .
$$

Comparing this with the relative $\operatorname{trace} \operatorname{tr}_{E}$ for $E=E(\nu)$, it follows immediately for all $M \in \mathcal{M}^{\infty}(\chi(\tau))$ that

$$
\operatorname{adj}_{M}^{2} \circ \operatorname{adj}_{M}^{1}=\operatorname{tr}_{E(\nu)}^{M}\left(\operatorname{pr}_{\chi(\tau+\nu)}\right) \in \operatorname{End}_{\mathfrak{g}}(M)
$$

or, regarded as a natural transformation of the identity functor Id : $\mathcal{M}^{\infty}(\chi(\tau))$ $\rightarrow \mathcal{M}^{\infty}(\chi(\tau))$ to itself

$$
\operatorname{adj}^{2} \circ \operatorname{adj}^{1}=\operatorname{tr}_{E(\nu)}\left(\operatorname{pr}_{\chi(\tau+\nu)}\right) .
$$

Let now $\iota_{\chi}: \mathcal{M}(\chi) \hookrightarrow \mathcal{M}$ denote the embedding functor, let $F, G: \mathcal{M}^{\infty}(\chi) \rightarrow \mathcal{M}$ be functors and denote by $F(\chi)$, resp. $G(\chi)$ its restrictions to the subcategory $\mathcal{M}(\chi)$. Each natural transformation $n$ from $F$ to $G$ can be regarded as natural transformation from $F(\chi)$ to $G(\chi)$ by first applying $\iota_{\chi}$ and then $n$. In particular,

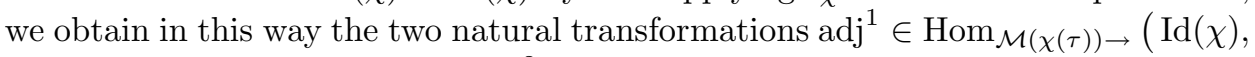
$\left.T_{\tau+\nu}^{\tau} \circ T_{\tau}^{\tau+\nu}(\chi)\right)$ and similarly $\operatorname{adj}^{2} \in \operatorname{Hom}_{\mathcal{M}(\chi(\tau)) \rightarrow}\left(T_{\tau+\nu}^{\tau} \circ T_{\tau}^{\tau+\nu}(\chi), \operatorname{Id}(\chi)\right)$.

5.3.3. We have $\Delta\left(-\nu, \nu ; w_{0}\right)(\tau)=\operatorname{adj}_{M\left(w_{0} \cdot \tau\right)}^{2} \circ \operatorname{adj}_{M\left(w_{0} \cdot \tau\right)}^{1}$. By choosing for $M$ the Verma module $M(\tau)$, we can assign to each $\tau \in \mathfrak{h}^{*}$ a canonical element $\operatorname{adj}_{M(\tau)}^{2} \circ$ $\operatorname{adj}_{M(\tau)}^{1} \in \operatorname{End}(M(\tau)) \cong k$. We will see in the following, that for dominant $\nu$ this is precisely the triangle function $\Delta\left(-\nu, \nu ; w_{0}\right)(\tau)$. For $x \in \mathcal{W}$ and $\tau$ generic we call $\phi_{x}(x \cdot \tau)$ the isomorphism $T_{\tau+\nu}^{\tau} T_{\tau}^{\tau+\nu} M(x \cdot \tau) \stackrel{\sim}{\longrightarrow} M(x \cdot \tau)$ given by $\left(\phi_{x}(x \cdot \tau)\right)^{-1}=$ $T_{\tau+\nu}^{\tau} F_{x \nu}(x \cdot \tau) \circ F_{-x \nu}(x \cdot(\tau+\nu))$ (see 4.3). First we show that for dominant $\nu$ and 
generic $\tau$ the following diagram commutes:

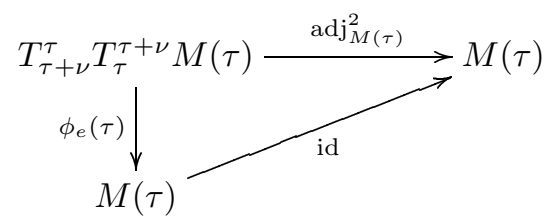

Let again denote $e_{\nu} \in E(\nu)_{\nu}$ the fixed extremal weight vector for a dominant integral weight $\nu$. Since $w_{0}$ is the longest element in the Weyl group, the dominance of $\nu$ implies that $e_{-\nu}:=\dot{w}_{0} e_{\nu}$ is a weight vector of weight $-\nu$. Here, $\dot{w}_{0} \in G$ is a representative of $w_{0} \in N_{G}(T) / T$.

Define now a pairing $E(-\nu) \times E(\nu) \rightarrow k$ by $\left\langle e_{-\nu}, e_{\nu}\right\rangle:=1$ and obtain thus an identification $\varphi: E(\nu)^{*} \stackrel{\sim}{\longrightarrow} E(-\nu)$. Let $v_{\tau} \in M(\tau)$ be the canonical generator. To see that the above diagram commutes, it suffices to show that the pre-image of $v_{\tau}$ in $T_{\tau+\nu}^{\tau} T_{\tau}^{\tau+\nu} M(\tau)$ is mapped again to $v_{\tau}$ when applying $\operatorname{adj}_{M(\tau)}^{2}$. We have

$$
\begin{aligned}
\left(\phi_{e}(\tau)\right)^{-1}\left(v_{\tau}\right) & =\operatorname{pr}_{\chi(\tau)}\left(e_{-\nu} \otimes\left(\operatorname{pr}_{\chi(\tau+\nu)}\left(e_{\nu} \otimes v_{\tau}\right)\right)\right) \\
& =\operatorname{pr}_{\chi(\tau)}\left(e_{-\nu} \otimes e_{\nu} \otimes v_{\tau}\right) \\
& =e_{-\nu} \otimes e_{\nu} \otimes v_{\tau}-\bigoplus_{\chi \neq \chi(\tau)} \operatorname{pr}_{\chi}\left(e_{-\nu} \otimes e_{\nu} \otimes v_{\tau}\right) .
\end{aligned}
$$

The first equation holds by definition of $\phi_{e}(\tau)$, the second follows since for dominant $\nu$ we have $\operatorname{pr}_{\chi(\tau+\nu)}\left(e_{\nu} \otimes v_{\tau}\right)=e_{\nu} \otimes v_{\tau}$ (see the example in 3.1) and the third equation is just a direct sum decomposition. Since $\operatorname{adj}_{M}^{2}$ was the composition

$$
T_{\tau+\nu}^{\tau} T_{\tau}^{\tau+\nu} M \stackrel{\varphi^{-1} \otimes \mathrm{pr}}{\longrightarrow} E^{*} \otimes E \otimes M \stackrel{\text { cont } \otimes \operatorname{id}_{M}}{\longrightarrow} M
$$

we know in particular that $\operatorname{adj}_{M(\tau)}^{2}$ is a $\mathfrak{g}$-module homomorphism to $M(\tau)$. Then it is clear, that $\operatorname{adj}_{M(\tau)}^{2}$ maps $\bigoplus_{\chi \neq \chi(\tau)} \operatorname{pr}_{\chi}\left(e_{-\nu} \otimes e_{\nu} \otimes v_{\tau}\right)$ to zero since this element has the wrong central character. For $e_{-\nu} \otimes e_{\nu} \otimes v_{\tau}$ we use the fact that $\left\langle e_{-\nu}, e_{\nu}\right\rangle=1$ and obtain as an image under $\operatorname{adj}_{M(\tau)}^{2}$ precisely $v_{\tau}$. Taken together, we get $\operatorname{adj}_{M(\tau)}^{2}=$ id $\circ \phi_{e}(\tau)$, i.e. the above diagram commutes.

This now means that under the map $\operatorname{Nat}(-\nu, \nu ; e)(\tau)$ the image of $\operatorname{adj}^{2} \in$

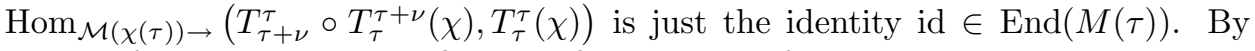
means of the uncanonical definition of the triangle function we deduce

$$
\begin{aligned}
\Delta\left(-\nu, \nu ; w_{0}\right)(\tau) & =\operatorname{Nat}\left(-\nu, \nu ; w_{0}\right)(\tau) \circ(\operatorname{Nat}(-\nu, \nu ; e)(\tau))^{-1}\left(\operatorname{id}_{M(\tau)}\right) \\
& =\left(\operatorname{Nat}\left(-\nu, \nu ; w_{0}\right)(\tau)\right)\left(\operatorname{adj}^{2}\right)
\end{aligned}
$$

and the Theorem of Bernstein-Gelfand implies that the right hand side of the following diagram commutes:

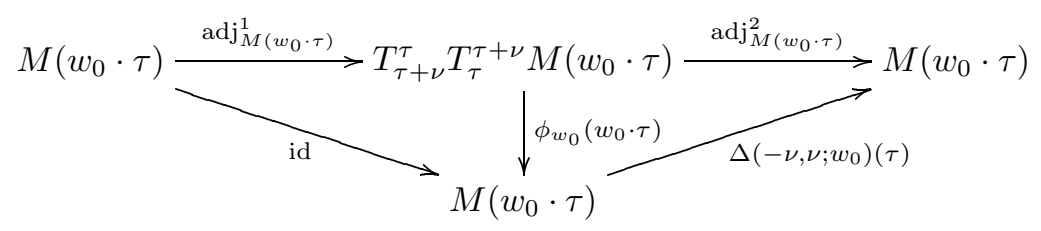


The commutativity of the left hand side can be shown in an analogous way. We thus obtain for dominant $\nu$

$$
\operatorname{adj}_{M\left(w_{0} \cdot \tau\right)}^{2} \circ \operatorname{adj}_{M\left(w_{0} \cdot \tau\right)}^{1}=\Delta\left(-\nu, \nu ; w_{0}\right)(\tau)
$$

and together with the equation $\operatorname{adj}_{M\left(w_{0} \cdot \tau\right)}^{2} \circ \operatorname{adj}_{M\left(w_{0} \cdot \tau\right)}^{1}=\operatorname{tr}_{E(\nu)}^{M\left(w_{0} \cdot \tau\right)}\left(\operatorname{pr}_{\chi(\tau+\nu)}\right)$ from section 5.3.2 we deduce Theorem 2 .

5.4. The case $M(\tau) \longrightarrow T_{\tau+\nu}^{\tau} T_{\tau}^{\tau+\nu} M(\tau) \longrightarrow M(\tau)$.

Corollary of the proof. Let $\nu \in P^{+}$be dominant and $\tau \in \mathfrak{h}^{*}$ generic. Then the composition

$$
M(\tau) \stackrel{\operatorname{adj}_{M(\tau)}^{1}}{\longrightarrow} T_{\tau+\nu}^{\tau} T_{\tau}^{\tau+\nu} M(\tau) \stackrel{\operatorname{adj}_{M(\tau)}^{2}}{\longrightarrow} M(\tau)
$$

is just multiplication with $s(\tau):=\prod_{\alpha \in R^{+}} \frac{\left\langle\tau+\nu+\rho, \alpha^{\vee}\right\rangle}{\left\langle\tau+\rho, \alpha^{\vee}\right\rangle}$, where $s$ is considered as an element in $\operatorname{Quot}(S(\mathfrak{h}))$.

Proof. The commutativity of the diagram $(*)$ in the proof of Theorem 2 is equivalent to the commutativity of

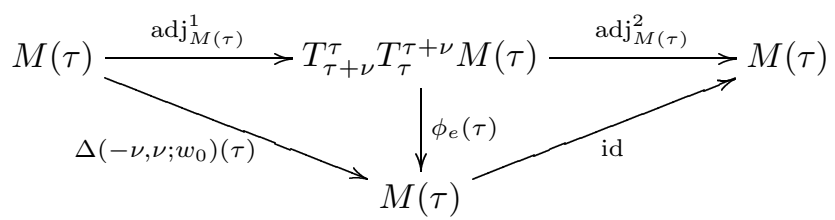

Hence $\left(\operatorname{adj}^{2} \circ \operatorname{adj}^{1}\right)_{M(\tau)}=\Delta\left(-\nu, \nu ; w_{0}\right)(\tau)=\prod_{\alpha \in R^{+}} \frac{\left\langle\tau+\nu+\rho, \alpha^{\vee}\right\rangle}{\left\langle\tau+\rho, \alpha^{\vee}\right\rangle}=s(\tau)$.

\section{Calculation of $\Delta$}

Theorem 3. For $\lambda \in \mathfrak{h}^{*}$ set $\bar{\alpha}(\lambda):=1$ if $\left\langle\lambda, \alpha^{\vee}\right\rangle\left\langle 0\right.$ and $\bar{\alpha}(\lambda):=0$ if $\left\langle\lambda, \alpha^{\vee}\right\rangle \geq 0$. Let $\nu, \mu \in P$ be integral weights and $x \in \mathcal{W}$. Then there exists a constant $c \in k^{\times}$, independent of $\tau, \nu, \mu$ and $x$, such that

$$
\Delta(\mu, \nu ; x)(\tau-\rho)=c \prod_{\substack{\alpha \in R^{+} \\ \text {with } x \alpha \in R^{-}}} \frac{\left\langle\tau, \alpha^{\vee}\right\rangle^{\bar{\alpha}(\nu)}\left\langle\tau+\nu, \alpha^{\vee}\right\rangle^{\bar{\alpha}(\mu)}\left\langle\tau+\nu+\mu, \alpha^{\vee}\right\rangle^{\bar{\alpha}(\nu+\mu)}}{\left\langle\tau, \alpha^{\vee}\right\rangle^{\bar{\alpha}(\nu+\mu)}\left\langle\tau+\nu, \alpha^{\vee}\right\rangle^{\bar{\alpha}(\nu)}\left\langle\tau+\nu+\mu, \alpha^{\vee}\right\rangle^{\bar{\alpha}(\mu)}}
$$

Proof. Postponed.

For an integral weight $\nu \in P$ define the map $\delta_{\nu} \in \mathcal{P}\left(\mathfrak{h}^{*}\right)$ as in Section 3.2 by

$$
\delta_{\nu}(\tau):=\prod_{\alpha \in R^{+}} \prod_{0 \leq n_{\alpha}<-\left\langle\nu, \alpha^{\vee}\right\rangle}\left(\left\langle\tau+\rho, \alpha^{\vee}\right\rangle-n_{\alpha}\right) .
$$

Lemma 11. Let $\pi(\tau)$ be the product on the right hand side of the equation in Theorem 3. Then

$$
\pi(\tau+\rho)= \pm \frac{\delta_{\nu}(\tau) \delta_{\mu}(\tau+\nu) \delta_{x(\nu+\mu)}(x \cdot \tau)}{\delta_{x \nu}(x \cdot \tau) \delta_{x \mu}(x \cdot(\tau+\nu)) \delta_{\nu+\mu}(\tau)}
$$


Proof. Let $x \in \mathcal{W}$ be fixed and let $\alpha \in R^{+}$be a positive root. We then have in $\delta_{\nu}(\tau)$ the factor $\prod_{0 \leq n<-\left\langle\nu, \alpha^{\vee}\right\rangle}\left(\left\langle\tau+\rho, \alpha^{\vee}\right\rangle-n\right)$. Suppose now that $x \alpha$ is also a positive root. Then we obtain in $\delta_{x \nu}(x \cdot \tau)$ the factor

$$
\begin{aligned}
\prod_{0 \leq n<-\left\langle x \nu, x \alpha^{\vee}\right\rangle}\left(\left\langle x \cdot \tau+\rho, x \alpha^{\vee}\right\rangle-n\right) & =\prod_{0 \leq n<-\left\langle\nu, \alpha^{\vee}\right\rangle}\left(\left\langle x(\tau+\rho), x \alpha^{\vee}\right\rangle-n\right) \\
& =\prod_{0 \leq n<-\left\langle\nu, \alpha^{\vee}\right\rangle}\left(\left\langle\tau+\rho, \alpha^{\vee}\right\rangle-n\right)
\end{aligned}
$$

and hence in the quotient $\delta_{\nu}(\tau) / \delta_{x \nu}(x \cdot \tau)$ all the products over $\alpha \in R^{+}$with $x \alpha \in R^{+}$cancel out. Let now $\alpha \in R^{+}$such that $x \alpha \notin R^{+}$, i.e. $-x \alpha$ is a positive root. In this case we have in $\delta_{x \nu}(x \cdot \tau)$ the factor

$$
\begin{aligned}
\prod_{0 \leq n<-\left\langle x \nu,-x \alpha^{\vee}\right\rangle}\left(\left\langle x \cdot \tau+\rho,-x \alpha^{\vee}\right\rangle-n\right) & =\prod_{0 \leq n<\left\langle\nu, \alpha^{\vee}\right\rangle}\left(-\left\langle\tau+\rho, \alpha^{\vee}\right\rangle-n\right) \\
& =(-1)^{\left\langle\nu, \alpha^{\vee}\right\rangle} \prod_{0 \leq n<\left\langle\nu, \alpha^{\vee}\right\rangle}\left(\left\langle\tau+\rho, \alpha^{\vee}\right\rangle+n\right)
\end{aligned}
$$

and taken together we get

$$
\frac{\delta_{\nu}(\tau)}{\delta_{x \nu}(x \cdot \tau)}=\prod_{\substack{\alpha \in R^{+} \\ \text {with } x \in R^{-}}}(-1)^{\left\langle\nu, \alpha^{\vee}\right\rangle} \frac{\prod_{0 \leq n_{\alpha}<-\left\langle\nu, \alpha^{\vee}\right\rangle}\left(\left\langle\tau+\rho, \alpha^{\vee}\right\rangle-n_{\alpha}\right)}{\prod_{0 \leq n_{\alpha}<\left\langle\nu, \alpha^{\vee}\right\rangle}\left(\left\langle\tau+\rho, \alpha^{\vee}\right\rangle+n_{\alpha}\right)} .
$$

Considering then the different cases according to whether $\left\langle\nu, \alpha^{\vee}\right\rangle,\left\langle\mu, \alpha^{\vee}\right\rangle$ and $\left\langle\nu+\mu, \alpha^{\vee}\right\rangle$ are positive or negative, we obtain the closed formula

$$
\frac{\delta_{\nu}(\tau) \delta_{\mu}(\tau+\nu) \delta_{x(\nu+\mu)}(x \cdot \tau)}{\delta_{x \nu}(x \cdot \tau) \delta_{x \mu}(x \cdot(\tau+\nu)) \delta_{\nu+\mu}(\tau)}=\varepsilon \pi(\tau+\rho),
$$

where $\varepsilon:=\prod_{\substack{\alpha \in R^{+} \\ \text {with } x \alpha \in R^{-}}}(-1)^{\left(\bar{\alpha}(\nu)\left\langle\nu, \alpha^{\vee}\right\rangle+\bar{\alpha}(\mu)\left\langle\mu, \alpha^{\vee}\right\rangle-\bar{\alpha}(\nu+\mu)\left\langle\mu+\nu, \alpha^{\vee}\right\rangle\right)}= \pm 1$.

Now, let $\tau \in \mathfrak{h}^{*}$ be a generic weight and recall (see 4.3) the map $\operatorname{Nat}_{x}(\tau):=$ $\operatorname{Nat}(\mu, \nu ; x)(\tau)$ :

$$
\begin{aligned}
\operatorname{Hom}_{\mathcal{M}(\chi(\tau))} & \rightarrow\left(T_{\tau+\nu}^{\tau+\nu+\mu} \circ T_{\tau}^{\tau+\nu}, T_{\tau}^{\tau+\nu+\mu}\right) \\
& \stackrel{\sim}{\longrightarrow} \operatorname{Hom}_{\mathfrak{g}}\left(T_{\tau+\nu}^{\tau+\nu+\mu} T_{\tau}^{\tau+\nu} M(x \cdot \tau), T_{\tau}^{\tau+\nu+\mu} M(x \cdot \tau)\right) \\
& \stackrel{\sim}{\longrightarrow} \operatorname{Hom}_{\mathfrak{g}}(M(x \cdot(\tau+\nu+\mu)), M(x \cdot(\tau+\nu+\mu))) \\
& \stackrel{\sim}{\longrightarrow} k .
\end{aligned}
$$

Denote the pre-image of $1 \in k$ under this isomorphism by the natural transformation $G^{x}(\tau):=\left(\operatorname{Nat}_{x}(\tau)\right)^{-1}(1) \in \operatorname{Hom}_{\mathcal{M}(\chi(\tau)) \rightarrow}\left(T_{\tau+\nu}^{\tau+\nu+\mu} \circ T_{\tau}^{\tau+\nu}, T_{\tau}^{\tau+\nu+\mu}\right)$.

Lemma 12. For generic $\tau \in \mathfrak{h}^{*}$ we have $G^{e}(\tau)=\Delta(\mu, \nu ; x)(\tau) G^{x}(\tau)$.

Proof. By definition, $\Delta(\mu, \nu ; x)(\tau)=\operatorname{Nat}_{x}(\tau) \circ\left(\operatorname{Nat}_{e}(\tau)\right)^{-1}(1)$ and therefore

$$
\begin{aligned}
G^{e}(\tau) & =\left(\operatorname{Nat}_{x}(\tau)\right)^{-1} \circ \operatorname{Nat}_{x}(\tau) \circ\left(\operatorname{Nat}_{e}(\tau)\right)^{-1}(1) \\
& =\left(\operatorname{Nat}_{x}(\tau)\right)^{-1}(\Delta(\mu, \nu ; x)(\tau)) \\
& =\Delta(\mu, \nu ; x)(\tau)\left(\operatorname{Nat}_{x}(\tau)\right)^{-1}(1) \\
& =\Delta(\mu, \nu ; x) G^{x}(\tau) .
\end{aligned}
$$


Let now integral weights $\nu_{1}, \ldots, \nu_{n}$ be given such that $\sum_{i=1}^{n} \nu_{i}=0$. We set the translation functor $T\left(\nu_{i}\right):=T_{\tau+\nu_{1}+\cdots+\nu_{i-1}}^{\tau+\nu_{1}+\cdots+\nu_{i}}$ when there is no ambiguity of the respective categories. For $\lambda \in P$ an integral weight and $\tau$ generic there are isomorphisms (see 4.3) $F_{x \lambda}(x \cdot \tau): M(x \cdot(\tau+\lambda)) \stackrel{\sim}{\longrightarrow} T_{\tau}^{\tau+\lambda} M(x \cdot \tau)$, such that $\left(F_{x \lambda}(x \cdot \tau)\right)\left(v_{x \cdot \tau}\right)=\operatorname{pr}_{\chi(\tau+\lambda)}\left(\dot{x} e_{\lambda} \otimes v_{x \cdot \tau}\right)$. Here, $e_{\lambda} \in E(\lambda)_{\lambda}$ is a fixed chosen extremal weight vector. Since $\sum_{i=1}^{n} \nu_{i}=0$, we can compose these isomorphisms to obtain an isomorphism $M(x \cdot \tau) \cong T\left(\nu_{n}\right) \cdots T\left(\nu_{1}\right) M(x \cdot \tau)$ and thus also

$$
\begin{aligned}
\operatorname{Hom}_{\mathfrak{g}}(M(x \cdot \tau), M(x \cdot \tau)) & \stackrel{\sim}{\longrightarrow} \operatorname{Hom}_{\mathfrak{g}}\left(M(x \cdot \tau), T\left(\nu_{n}\right) \cdots T\left(\nu_{1}\right) M(x \cdot \tau)\right) \\
& \hookrightarrow \operatorname{Hom}_{k}\left(\mathfrak{U}\left(\mathfrak{n}^{-}\right), E\left(\nu_{n}\right) \otimes \cdots \otimes E\left(\nu_{1}\right) \otimes \mathfrak{U}\left(\mathfrak{n}^{-}\right)\right),
\end{aligned}
$$

where we have identified the Verma module $M(x \cdot \tau)$ with $\mathfrak{U}\left(\mathfrak{n}^{-}\right)$. Call now the image of the identity on $M(x \cdot \tau)$ under this map $h^{x}\left(\nu_{1}, \ldots, \nu_{n}\right)(\tau)$ and let $\mathcal{U} \subset \mathfrak{h}^{*}$ be the set of all generic weights. We then obtain for all $x \in \mathcal{W}$ a function

$$
\begin{aligned}
& h^{x}\left(\nu_{1}, \ldots, \nu_{n}\right): \mathcal{U} \rightarrow \operatorname{Hom}_{k}\left(\mathfrak{U}\left(\mathfrak{n}^{-}\right), E\left(\nu_{n}\right) \otimes \cdots \otimes E\left(\nu_{1}\right) \otimes \mathfrak{U}\left(\mathfrak{n}^{-}\right)\right) \\
& \tau \mapsto \quad h^{x}\left(\nu_{1}, \ldots, \nu_{n}\right)(\tau) \text {, }
\end{aligned}
$$

such that $h^{x}\left(\nu_{1}, \ldots, \nu_{n}\right)(\tau)$ maps the element $v_{x \cdot \tau} \in M(x \cdot \tau) \cong \mathfrak{U}\left(\mathfrak{n}^{-}\right)$to the element $\operatorname{pr}_{\chi\left(\tau+\nu_{1}+\cdots+\nu_{n}\right)}\left(\dot{x} e_{\nu_{n}} \otimes\left(\cdots \otimes \operatorname{pr}_{\chi\left(\tau+\nu_{1}\right)}\left(\dot{x} e_{\nu_{1}} \otimes v_{x \cdot \tau}\right)\right) \cdots\right)$ for fixed vectors $e_{\nu_{i}} \in E\left(\nu_{i}\right)_{\nu_{i}}$.

Lemma 13. Set $d^{x}\left(\nu_{1}, \ldots, \nu_{n}\right)(\tau):=\delta_{x \nu_{1}}(x \cdot \tau) \delta_{x \nu_{2}}\left(x \cdot\left(\tau+\nu_{1}\right)\right) \cdots \delta_{x \nu_{n}}(x \cdot(\tau+$ $\left.\left.\nu_{1}+\cdots+\nu_{n-1}\right)\right)$. Then the map $d^{x}\left(\nu_{1}, \ldots, \nu_{n}\right) h^{x}\left(\nu_{1}, \ldots, \nu_{n}\right)$ is algebraic on $\mathcal{U}$ and there exists an algebraic extension on $\mathfrak{h}^{*}$ whose set of zeros has codimension $\geq 2$.

Remark. Here, we call a map $a: \mathcal{U} \rightarrow W$ to a vector space $W$ algebraic, if it is a morphism of varieties. Of course, this is defined only if $\operatorname{dim} W<\infty$. In our case however, the image of $h^{x}\left(\nu_{1}, \ldots, \nu_{n}\right)$ is always contained in a finite-dimensional subspace of $\operatorname{Hom}_{k}\left(\mathfrak{U}\left(\mathfrak{n}^{-}\right), E(\nu) \otimes \cdots \otimes E\left(\nu_{1}\right) \otimes \mathfrak{U}\left(\mathfrak{n}^{-}\right)\right)$and we may thus regard $h^{x}\left(\nu_{1}, \ldots, \nu_{n}\right)$ as a map between varieties.

Proof. Let $\nu \in P$ be an integral weight and recall the maps

$$
\begin{array}{cccc}
f_{\nu}: \mathfrak{h}^{*} & \longrightarrow & E(\nu) \otimes M(\tau) \cong E(\nu) \otimes \mathfrak{U}\left(\mathfrak{n}^{-}\right) \\
\tau & \mapsto & \operatorname{pr}_{\chi(\tau+\nu)}\left(e_{\nu} \otimes v_{\tau}\right) .
\end{array}
$$

Let now $x \in \mathcal{W}$ be fixed. For generic $\tau$ define then the map $a_{\nu}^{x}(\tau)$ by

$$
\begin{array}{ccc}
a_{\nu}^{x}(\tau): \quad M(x \cdot \tau) \cong \mathfrak{U}\left(\mathfrak{n}^{-}\right) & \longrightarrow & E(\nu) \otimes M(x \cdot \tau) \cong E(\nu) \otimes \mathfrak{U}\left(\mathfrak{n}^{-}\right) \\
v_{x \cdot \tau} & \mapsto & \delta_{x \nu}(x \cdot \tau) f_{x \nu}(x \cdot \tau)
\end{array}
$$

where $f_{x \nu}(x \cdot \tau)=\operatorname{pr}_{\chi(\tau+\nu)}\left(\dot{x} e_{\nu} \otimes v_{x \cdot \tau}\right)$. Since $f_{\nu}$ is algebraic on $\mathcal{U}$ (see Theorem $1)$, we obtain in this way also an algebraic map

$$
\begin{array}{ccc}
a_{\nu}^{x}: \mathcal{U} & \longrightarrow & \operatorname{Hom}_{k}\left(\mathfrak{U}\left(\mathfrak{n}^{-}\right), E(\nu) \otimes \mathfrak{U}\left(\mathfrak{n}^{-}\right)\right) \\
\tau & \mapsto & a_{\nu}^{x}(\tau) .
\end{array}
$$

Here again, the image of $a_{\nu}^{x}$ is contained in a finite-dimensional subspace of $\operatorname{Hom}_{k}\left(\mathfrak{U}\left(\mathfrak{n}^{-}\right), E(\nu) \otimes \mathfrak{U}\left(\mathfrak{n}^{-}\right)\right)$and we regard $a_{\nu}^{x}$ in this way as a map between varieties.

According to Theorem 1 there is an algebraic extension of $\delta_{\nu} f_{\nu}$ on $\mathfrak{h}^{*}$, which vanishes only on a set of codimension $\geq 2$. Therefore, also $a_{\nu}^{x}$ has such an algebraic extension and we call it again $a_{\nu}^{x}$. For generic $\tau$ we then have $\left(a_{\nu}^{x}(\tau)\right)\left(v_{x \cdot \tau}\right)=$ 
$\delta_{x \nu}(x \cdot \tau) \operatorname{pr}_{\chi(\tau+\nu)}\left(\dot{x} e_{\nu} \otimes v_{x \cdot \tau}\right)$. Note that for all $\tau \in \mathfrak{h}^{*}$ the vector $\left(a_{\nu}^{x}(\tau)\right)\left(v_{x \cdot \tau}\right) \in$ $E(\nu) \otimes M(x \cdot \tau)$ generates a Verma module with highest weight $x \cdot(\tau+\nu)$. The image of $a_{\nu}^{x}(\tau)$ is thus always contained in a Verma module $M(x \cdot(\tau+\nu)) \subset E(\nu) \otimes$ $M(x \cdot \tau)$ and we can identify the element $\left(a_{\nu}^{x}(\tau)\right)\left(v_{x \cdot \tau}\right)$ with the canonical generator $v_{x \cdot(\tau+\nu)} \in M(x \cdot(\tau+\nu))$. Using the isomorphism $M(x \cdot(\tau+\nu)) \cong \mathfrak{U}\left(\mathfrak{n}^{-}\right)$, we may then apply the map $a_{\nu^{\prime}}^{x}(\tau+\nu)$ for another weight $\nu^{\prime} \in P$. In particular, we can concatenate the maps $a_{\nu_{n}}^{x}\left(\tau+\nu_{1}+\cdots+\nu_{n-1}\right) \circ \cdots \circ a_{\nu_{2}}^{x}\left(\tau+\nu_{1}\right) \circ a_{\nu_{1}}^{x}(\tau)$ and obtain in this way an algebraic map

$$
\begin{aligned}
a: \quad \mathfrak{h}^{*} & \longrightarrow \quad \operatorname{Hom}_{k}\left(\mathfrak{U}\left(\mathfrak{n}^{-}\right), E\left(\nu_{n}\right) \otimes \cdots \otimes E\left(\nu_{1}\right) \otimes \mathfrak{U}\left(\mathfrak{n}^{-}\right)\right) \\
\tau & \mapsto a_{\nu_{n}}^{x}\left(\tau+\nu_{1}+\cdots+\nu_{n-1}\right) \circ \cdots \circ a_{\nu_{2}}^{x}\left(\tau+\nu_{1}\right) \circ a_{\nu_{1}}^{x}(\tau)
\end{aligned}
$$

which vanishes only on a set of codimension $\geq 2$ and which maps a generic weight $\tau$ to $a(\tau)=\delta_{x \nu_{1}}(x \cdot \tau) \delta_{x \nu_{2}}\left(x \cdot\left(\tau+\nu_{1}\right)\right) \cdots \delta_{x \nu_{n}}\left(x \cdot\left(\tau+\nu_{1}+\cdots \nu_{n-1}\right)\right) h^{x}\left(\nu_{1}, \ldots, \nu_{n}\right)(\tau)=$ $d^{x}\left(\nu_{1}, \ldots, \nu_{n}\right)(\tau) h^{x}\left(\nu_{1}, \ldots, \nu_{n}\right)(\tau)$. We thus obtain the map $a$ as the desired algebraic extension.

According to the Theorem of Bernstein-Gelfand we may interpret for generic $\tau$ the maps

$$
\begin{aligned}
h^{x}\left(\nu_{1}, \ldots, \nu_{n}\right)(\tau) & \in \operatorname{Hom}_{\mathfrak{g}}\left(M(x \cdot \tau), T\left(\nu_{n}\right) \cdots T\left(\nu_{1}\right) M(x \cdot \tau)\right) \\
& \cong \operatorname{Hom}_{\mathcal{M}(\chi(\tau)) \rightarrow\left(\operatorname{Id}, T\left(\nu_{n}\right) \cdots T\left(\nu_{1}\right)\right)}
\end{aligned}
$$

as natural transformations of functors. Set now

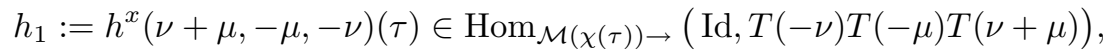

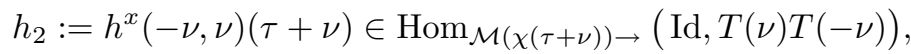

$$
\begin{aligned}
& h_{3}:=h^{x}(-\mu, \mu)(\tau+\nu+\mu) \in \operatorname{Hom}_{\mathcal{M}(\chi(\tau+\nu+\mu)) \rightarrow(\mathrm{Id}, T(\mu) T(-\mu))}
\end{aligned}
$$

and consider the natural transformations

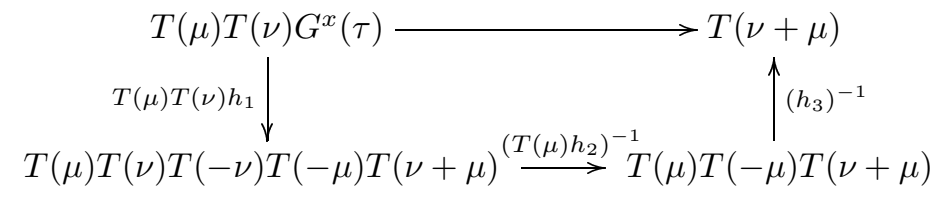

The diagram commutes, since $\left(h_{3}\right)^{-1} \circ\left(T(\mu) h_{2}\right)^{-1} \circ T(\mu) T(\nu) h_{1}$ as well as $G^{x}(\tau)$ imply the identity on $M(x \cdot(\tau+\nu+\mu))$ under the isomorphism

$\operatorname{Hom}_{\mathcal{M}(\chi(\tau)) \rightarrow(T(\mu) \circ T(\nu), T(\nu+\mu))}$

$$
\begin{aligned}
& \stackrel{\sim}{\longrightarrow} \operatorname{Hom}_{\mathfrak{g}}(T(\mu) T(\nu) M(x \cdot \tau), T(\nu+\mu) M(x \cdot \tau)) \\
& \stackrel{\sim}{\longrightarrow} \operatorname{Hom}_{\mathfrak{g}}(M(x \cdot(\tau+\nu+\mu)), M(x \cdot(\tau+\nu+\mu))) .
\end{aligned}
$$

Lemma 14. Set $D^{x}(\tau):=\delta_{x(\nu+\mu)}(x \cdot \tau) / \delta_{x \nu}(x \cdot \tau) \delta_{x \mu}(x \cdot(\tau+\nu))$. Then the map $D^{x} G^{x}$ is algebraic on $\mathcal{U}$ and there exists an algebraic extension on $\mathfrak{h}^{*}$ whose set of zeros has codimension $\geq 2$.

Proof. Since the maps $d^{x}\left(\nu_{1}, \ldots, \nu_{n}\right) h^{x}\left(\nu_{1}, \ldots, \nu_{n}\right)$ have such algebraic extensions (Lemma 13), the commutativity of the above diagram implies that also $D^{x} G^{x}$ has such an algebraic extension, where

$$
\begin{aligned}
D^{x}(\tau) & =d^{x}(\nu+\mu,-\mu,-\nu)(\tau) d^{x}(-\nu, \nu)(\tau+\nu) d^{x}(-\mu, \mu)(\tau+\nu+\mu) \\
& =\delta_{x(\nu+\mu)}(x \cdot \tau) / \delta_{x \nu}(x \cdot \tau) \delta_{x \mu}(x \cdot(\tau+\nu))
\end{aligned}
$$


Finally, we come to the following:

Proof of Theorem 3. By Lemma 11 it suffices to show that $\Delta(\mu, \nu ; x)(\tau)=$ $c \pi(\tau+\rho)$ for a non-vanishing constant $c$. Note, that $\left(D^{x} / D^{e}\right)(\tau)= \pm \pi(\tau+\rho)$. We deduce from Lemma 12 that for generic weights $D^{x} D^{e} G^{e}=\Delta(\mu, \nu ; x) D^{e} D^{x} G^{x}$. Since $D^{e} G^{e}$ as well as $D^{x} G^{x}$ have algebraic extensions on $\mathfrak{h}^{*}$ which vanish only on a set of codimension $\geq 2$ (Lemma 14), it follows that there is a constant $c \in k^{\times}$, independent of $\tau, \mu, \nu$ and $x$, such that

$$
c \Delta(\mu, \nu ; x)(\tau)=\left(D^{x} / D^{e}\right)(\tau)= \pm \pi(\tau+\rho) .
$$

\section{OutLOOK}

7.1. Identities. There are many nice identities for the triangle functions. Obvious are the

\section{Normalization identities.}

$$
\Delta(\mu, \nu ; e)=1 \quad \Delta(0, \nu ; x)=1 \quad \Delta(\nu, 0 ; x)=1 .
$$

By means of Theorem 3 or directly with the definition of $\Delta$ one then checks for $\nu, \mu, \eta \in P$ and $x, y \in \mathcal{W}$ :

\section{Decomposition identity.}

$$
\Delta(\eta+\mu, \nu ; x)(\tau) \Delta(\eta, \mu ; x)(\tau+\nu)=\Delta(\eta, \mu+\nu ; x)(\tau) \Delta(\mu, \nu ; x)(\tau) .
$$

\section{Rotation identity.}

$$
\Delta(y \mu, y \nu ; x)(y \cdot \tau)=(\Delta(\mu, \nu ; y)(\tau))^{-1} \Delta(\mu, \nu ; x y)(\tau) .
$$

Flat triangle identity. Let $\nu, \mu \in P$ be in the closure of a Weyl chamber. Then $\Delta(\mu, \nu ; x)=1$ for all $x \in \mathcal{W}$.

\subsection{Generalizations.}

7.2.1. The Weyl group parameter. Let $\nu, \mu \in P$ and $x, y \in \mathcal{W}$. Instead of applying the translation functors to the Verma modules $M(\tau)$ and $M(x \cdot \tau)$, we may choose the Verma modules $M(x \cdot \tau)$ and $M(y \cdot \tau)$. We then define a generalized triangle function $\Delta_{\mathrm{g}}$ by

$$
\Delta_{\mathrm{g}}(\mu, \nu ; y, x)(\tau):=\operatorname{det}\left(y x^{-1} \circ \operatorname{nat}(\mu, \nu ; x)(\tau) \circ(\operatorname{nat}(\mu, \nu ; y)(\tau))^{-1}\right) .
$$

We now have $\Delta_{\mathrm{g}}(\mu, \nu ; e, x)(\tau)=\Delta(\mu, \nu ; x)(\tau)$ and going back to the definition of $\Delta_{\mathrm{g}}$ we deduce the identities

(I1) $\Delta_{\mathrm{g}}(\mu, \nu ; y, x)(\tau)=(\Delta(\mu, \nu ; y)(\tau))^{-1} \Delta(\mu, \nu ; x)(\tau)$,

(I2) $\Delta_{\mathrm{g}}(\mu, \nu ; y, x)(\tau)=\left(\Delta_{\mathrm{g}}(\mu, \nu ; x, y)(\tau)\right)^{-1}$

and

(I3) $\Delta_{\mathrm{g}}(\mu, \nu ; y, x) \Delta_{\mathrm{g}}(\mu, \nu ; x, z)=\Delta_{\mathrm{g}}(\mu, \nu ; y, z)$.

The equivalent statement to the Rotation identity is obtained by comparing (I1) with

(I4) $\Delta_{\mathrm{g}}(\mu, \nu ; y, x)(\tau)=\Delta\left(y \mu, y \nu ; x y^{-1}\right)(y \cdot \tau)$. 
7.2.2. Number of translations. The triangle functions measure in a subtle way the relation between the two translation functors $T_{\tau+\nu}^{\tau+\nu+\mu} \circ T_{\tau}^{\tau+\nu}$ and $T_{\tau}^{\tau+\nu+\mu}$. Therefore the triangle

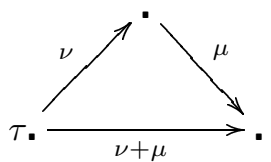

Let now integral weights $\nu_{1}, \ldots, \nu_{n}$ and $\mu_{1}, \ldots, \mu_{m} \in P$ be given such that $\sum_{i=1}^{n} \nu_{i}=\sum_{j=1}^{m} \mu_{j}=: p$. Then call $T\left(\nu_{i}\right)$ the translation functor

$$
\begin{array}{cl}
T\left(\nu_{i}\right): \mathcal{M}^{\infty}\left(\chi\left(\tau+\nu_{1}+\cdots+\nu_{i-1}\right)\right) & \longrightarrow \mathcal{M}^{\infty}\left(\chi\left(\tau+\nu_{1}+\cdots+\nu_{i}\right)\right) \\
M & \mapsto \operatorname{pr}_{\chi\left(\tau+\nu_{1}+\cdots+\nu_{i}\right)}\left(E\left(\nu_{i}\right) \otimes M\right)
\end{array}
$$

and define similarly the translation functor $T\left(\mu_{i}\right)$. We now want to compare the functors $T\left(\nu_{n}\right) \circ \cdots \circ T\left(\nu_{1}\right)$ and $T\left(\mu_{m}\right) \circ \cdots \circ T\left(\mu_{1}\right)$ with each other and instead of a triangle of translations we thus have now the following situation:

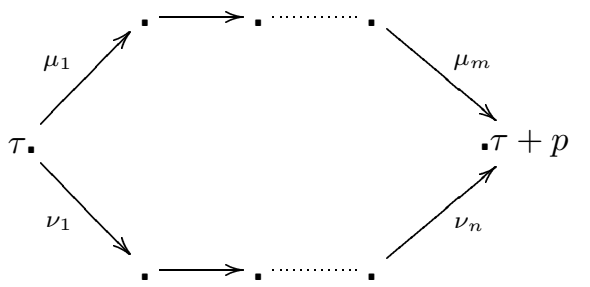

We start by defining a map năt $\left(\mu_{m}, \ldots, \mu_{1} ; \nu_{n}, \ldots, \nu_{1} ; x\right)(\tau)$ for $x \in \mathcal{W}$ and generic weight $\tau$ analogously to the definition of $\operatorname{nat}(\mu, \nu ; x)(\tau)$ as the composition

$$
\begin{aligned}
& \operatorname{Hom}_{\mathcal{M}}(\chi(\tau)) \rightarrow\left(T\left(\mu_{m}\right) \circ \cdots \circ T\left(\mu_{1}\right), T\left(\nu_{n}\right) \circ \cdots \circ T\left(\nu_{1}\right)\right) \\
& \quad \stackrel{\sim}{\longrightarrow} \operatorname{Hom}_{\mathfrak{g}}\left(T\left(\mu_{m}\right) \cdots T\left(\mu_{1}\right) M(x \cdot \tau), T\left(\nu_{n}\right) \cdots T\left(\nu_{1}\right) M(x \cdot \tau)\right) \\
& \quad \stackrel{\sim}{\longrightarrow} \operatorname{Hom}_{\mathfrak{g}}\left(E_{\mu_{m}} \hat{\otimes} \cdots \hat{\otimes} E_{\mu_{1}} \hat{\otimes} M(x \cdot(\tau+p)), E_{\nu_{n}} \hat{\otimes} \cdots \hat{\otimes} E_{\nu_{1}} \hat{\otimes} M(x \cdot(\tau+p))\right) \\
& \quad \stackrel{\sim}{\longrightarrow} E_{\mu_{m}}^{*} \otimes \cdots \otimes E_{\mu_{1}}^{*} \otimes E_{\nu_{n}} \otimes \cdots \otimes E_{\nu_{1}} .
\end{aligned}
$$

Here, we wrote $E_{\mu}$ for $E(\mu)_{x \mu}$. We then obtain a generalized triangle function $\diamond$ by

$$
\begin{aligned}
\diamond\left(\mu_{m}, \ldots \mu_{1} ; \nu_{n}, \ldots \nu_{1} ; x\right)(\tau):=\operatorname{det}\left(x^{-1}\right. & \circ \operatorname{nāt}\left(\mu_{m}, \ldots \mu_{1} ; \nu_{n}, \ldots \nu_{1} ; x\right)(\tau) \\
& \left.\circ\left(\operatorname{nāt}\left(\mu_{m}, \ldots \mu_{1} ; \nu_{n}, \ldots \nu_{1} ; e\right)(\tau)\right)^{-1}\right) .
\end{aligned}
$$

Obviously we have $\diamond(\mu, \nu ; \nu+\mu ; x)=\Delta(\mu, \nu ; x)$ as well as

$$
\diamond\left(\mu_{m}, \ldots, \mu_{1} ; \nu_{n}, \ldots, \nu_{1} ; x\right)(\tau)=\left(\diamond\left(\nu_{n}, \ldots, \nu_{1} ; \mu_{m}, \ldots, \mu_{1} ; x\right)(\tau)\right)^{-1}
$$

and we can reduce the calculation of $\diamond$ to the calculation of $\Delta$ by means of the

\section{Split identities.}

$$
\begin{aligned}
& \diamond\left(\nu_{3}, \nu_{2}, \nu_{1} ; \nu_{3}+\nu_{2}+\nu_{1} ; x\right)(\tau)=\Delta\left(\nu_{2}, \nu_{1} ; x\right)(\tau) \Delta\left(\nu_{3}, \nu_{1}+\nu_{2} ; x\right)(\tau) \\
&=\Delta\left(\nu_{2}+\nu_{3}, \nu_{1} ; x\right)(\tau) \Delta\left(\nu_{3}, \nu_{2} ; x\right)\left(\tau+\nu_{1}\right), \\
& \diamond\left(\mu_{2}, \mu_{1} ; \nu_{2}, \nu_{1} ; x\right)(\tau)=\Delta\left(\mu_{2}, \mu_{1} ; x\right)(\tau)\left(\Delta\left(\nu_{2}, \nu_{1} ; x\right)(\tau)\right)^{-1}
\end{aligned}
$$


The first identity just means that we can split

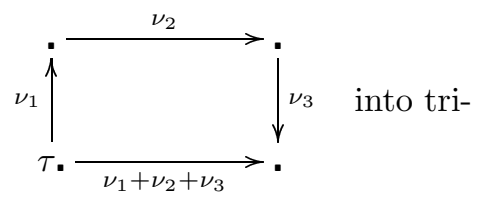

angles according to
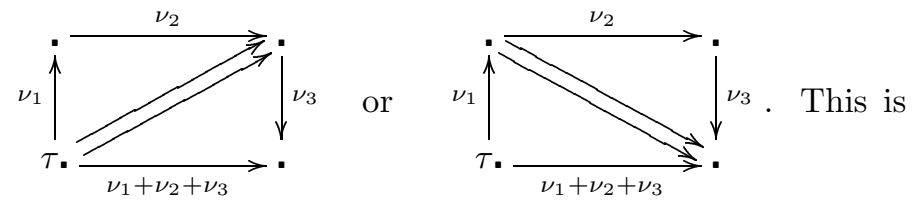

just the Decomposition identity in 7.1. The second equation describes the decom-

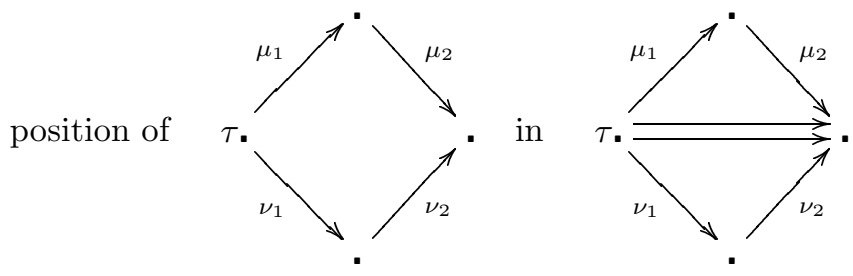

Inductively, we may thus first split up our situation into

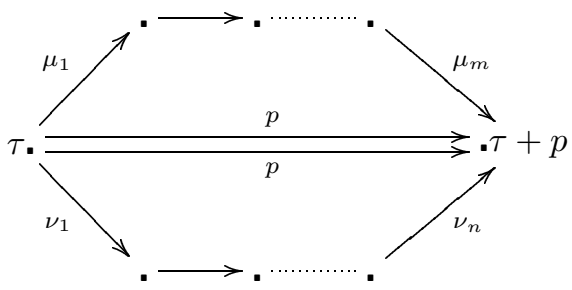

In formulae:

$$
\begin{aligned}
\diamond\left(\mu_{m}, \ldots \mu_{1} ; \nu_{n}, \ldots \nu_{1} ; x\right)(\tau) & =\diamond\left(\mu_{m}, \ldots \mu_{1} ; p ; x\right)(\tau) \diamond\left(p ; \nu_{n}, \ldots \nu_{1} ; x\right)(\tau) \\
& =\diamond\left(\mu_{m}, \ldots \mu_{1} ; p ; x\right)(\tau)\left(\diamond\left(\nu_{n}, \ldots \nu_{1} ; p ; x\right)(\tau)\right)^{-1}
\end{aligned}
$$

and in order to calculate the $\diamond$-functions it suffices thus to know them in the special case $\diamond\left(\mu_{m}, \ldots, \mu_{1} ; \sum_{j=1}^{m} \mu_{j} ; x\right)$. This situation can then be reduced to triangles by decomposing it into

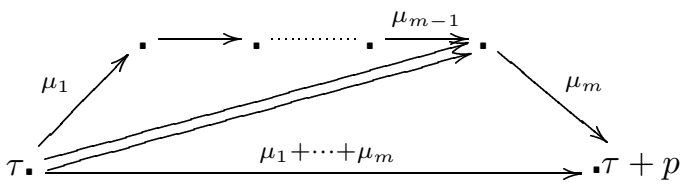

or into

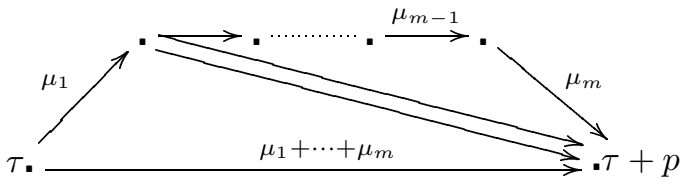

Inductively one can then prove 
Proposition. Let $\mu_{1}, \ldots, \mu_{m} \in P$ be integral weights and $x \in \mathcal{W}$. Then

$$
\begin{aligned}
\diamond\left(\mu_{m}, \ldots, \mu_{1} ; \sum_{j=1}^{m} \mu_{j} ; x\right)(\tau) & =\prod_{k=2}^{m} \Delta\left(\mu_{k}, \sum_{i=1}^{k-1} \mu_{i} ; x\right)(\tau) \\
& =\prod_{k=1}^{m-1} \Delta\left(\sum_{j=k+1}^{m} \mu_{j}, \mu_{k} ; x\right)\left(\tau+\sum_{j=1}^{k-1} \mu_{j}\right) .
\end{aligned}
$$

\section{ACKNOWLEDGMENT}

I am grateful to my advisor, Wolfgang Soergel, for his constant encouragement and all the fruitful discussions we had. Without his many ideas this work would not have been possible. I also want to thank Bernard Leclerc for bringing the article [Ka] to my attention.

\section{REFERENCES}

[AJS] Andersen, H.H., Jantzen, J.-C., Soergel, W.: Representations of quantum groups at a pth root of unity and of semisimple groups in characteristic $p$ : Independence of $p$. Astérisque 220 (1994), 1-320. MR 95j:20036

[Be] Bernstein, J.N.: Trace in Categories. In Operator Algebras, Unitary Representations, Enveloping Algebras and Invariant Theory (A. Connes, M. Duflo, A. Joseph, R. Rentschler, editors), Actes du colloque en l'honneur de Jacques Dixmier, Progress in Mathematics 92 Birkhäuser, 1990, 417-423. MR 92d:17010

[BG] Bernstein, J.N., Gelfand, S.I: Tensor products of finite and infinite dimensional representations of semisimple Lie algebras. Compositio Math. 41 (1980), 245-285. MR 82c:17003

[BGG1] Bernstein, J.N., Gelfand, I.M., Gelfand, S.I.: Structure of representations generated by vectors of highest weight. Funct. Anal. App. 5 (1971), 1-8. MR 45:298

[BGG2] _ Category of $\mathfrak{g}$-modules. Funct. Anal. App. 10 (1976), 87-92.

[Di] Dixmier, J.: Enveloping Algebras. (North Holland Mathematical Library, Vol. 14) Amsterdam-New York-Oxford: North Holland, 1977. MR 58:16803b

[ES] Etingof, P., Styrkas, K.: Algebraic integrability of Schrödinger operators and representations of Lie algebras. Compositio Math. 98 (1995), 91-112. MR 96j:58076

[EV] Etingof, P., Varchenko, A.: Exchange Dynamical Quantum Groups. On the xxx-preprint server under math.QA/9801135.

[HS] Hilton, J.P., Stammbach, U.: A course in homological algebra. New York-HeidelbergBerlin: Springer, 1971. MR 49:10751

[Ja1] Jantzen, J.C.: Moduln mit einem höchsten Gewicht. (Lecture Notes in Mathematics, Vol. 750) Berlin-Heidelberg-New York: Springer 1979. MR 81m:17011

[Ja2] _ Einhüllende Algebren halbeinfacher Lie-Algebren. Berlin-Heidelberg-New YorkTokyo: Springer 1983. MR 86c:17011

[Ka] Kashiwara, M.: The Universal Verma Module and the b-Function. In R. Hotta, editor, Algebraic Groups and Related Topics, Proceedings of Symposia Kyoto 1983, Nagoya, 1983, Adv. Stud. in Pure Mathematics 6, Kinokuniya Tokyo and North-Holland, Amsterdam, 1985, 67-81. MR 87i:22047

[Ko] Kostant, B.: On the Tensor Product of a Finite and an Infinite Dimensional Representation. Journal of Functional Analysis, 20 (1975), 257-285. MR 54:2888

[So] Soergel, W.: Kategorie $\mathcal{O}$, Perverse Garben und Moduln über den Koinvarianten zur Weylgruppe. Journal of the American Mathematical Society, Vol. 3, No. 2. April, 1990. MR 91e: 17007

Universität Freiburg, Mathematisches Institut, Eckerstr.1, D-79104 Freiburg, GerMANY

E-mail address: karen@mathematik.uni-freiburg.de 